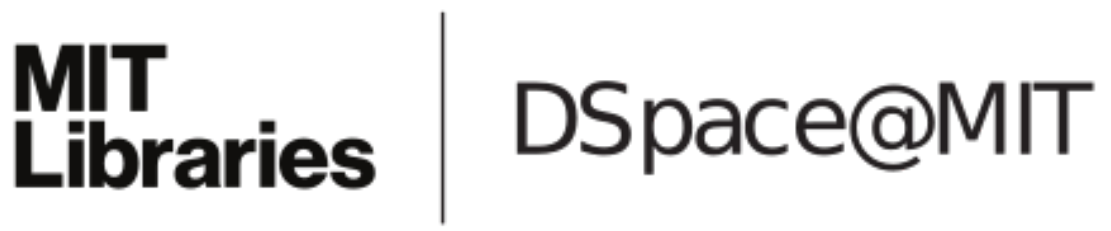

\author{
MIT Open Access Articles
}

\section{Fundamental Limits of Wideband Localization - Part II: Cooperative Networks}

The MIT Faculty has made this article openly available. Please share how this access benefits you. Your story matters.

Citation: Shen, Yuan, Henk Wymeersch, and Moe Z. Win. "Fundamental Limits of Wideband Localization Part II: Cooperative Networks." IEEE Transactions on Information Theory 56.10 (2010) : 4981-5000. (c) 2010 IEEE

As Published: http://dx.doi.org/10.1109/tit.2010.2059720

Publisher: Institute of Electrical and Electronics Engineers

Persistent URL: http://hdl.handle.net/1721.1/66247

Version: Final published version: final published article, as it appeared in a journal, conference proceedings, or other formally published context

Terms of Use: Article is made available in accordance with the publisher's policy and may be subject to US copyright law. Please refer to the publisher's site for terms of use. 


\title{
Fundamental Limits of Wideband Localization- Part II: Cooperative Networks
}

\author{
Yuan Shen, Student Member, IEEE, Henk Wymeersch, Member, IEEE, and Moe Z. Win, Fellow, IEEE
}

\begin{abstract}
The availability of position information is of great importance in many commercial, governmental, and military applications. Localization is commonly accomplished through the use of radio communication between mobile devices (agents) and fixed infrastructure (anchors). However, precise determination of agent positions is a challenging task, especially in harsh environments due to radio blockage or limited anchor deployment. In these situations, cooperation among agents can significantly improve localization accuracy and reduce localization outage probabilities. A general framework of analyzing the fundamental limits of wideband localization has been developed in Part $I$ of the paper. Here, we build on this framework and establish the fundamental limits of wideband cooperative location-aware networks. Our analysis is based on the waveforms received at the nodes, in conjunction with Fisher information inequality. We provide a geometrical interpretation of equivalent Fisher information (EFI) for cooperative networks. This approach allows us to succinctly derive fundamental performance limits and their scaling behaviors, and to treat anchors and agents in a unified way from the perspective of localization accuracy. Our results yield important insights into how and when cooperation is beneficial.
\end{abstract}

Index Terms-Cooperative localization, Cramér-Rao bound (CRB), equivalent Fisher information (EFI), information inequality, ranging information (RI), squared position error bound (SPEB).

\section{INTRODUCTION}

$\mathbf{T}$ HE availability of absolute or relative positional information is of great importance in many applications, such as localization services in cellular networks, search-and-rescue operations, asset tracking, blue force tracking, vehicle routing, and intruder detection [1]-[8]. Location-aware networks generally consist of two kinds of nodes: anchors and agents (see Fig. 1), where anchors have known positions while agents have

Manuscript received June 21, 2009; revised February 19, 2010. Date of current version September 15, 2010. This work was supported in part by the National Science Foundation under Grant ECCS-0901034, the Office of Naval Research Presidential Early Career Award for Scientists and Engineers (PECASE) N00014-09-1-0435, and the MIT Institute for Soldier Nanotechnologies. The material in this paper was presented in part at the IEEE Wireless Communications and Networking Conference, Hong Kong, March 2007 and the IEEE International Symposium on Spread Spectrum Techniques \& Applications, Bologna, Italy, August 2008.

Y. Shen and M. Z. Win are with the Laboratory for Information and Decision Systems (LIDS), Massachusetts Institute of Technology, Cambridge, MA 02139 USA (e-mail: shenyuan@mit.edu; moewin@mit.edu).

H. Wymeersch was with the Laboratory for Information and Decision Systems (LIDS), Massachusetts Institute of Technology, Cambridge, MA 02139 USA. He is now with the Department of Signals and Systems, Chalmers University of Technology, Gothenburg, Sweden (e-mail: henkw@ chalmers.se).

Communicated by M. Franceschetti, Associate Editor for Communication Networks.

Color versions of one or more of the figures in this paper are available online at http://ieeexplore.ieee.org.

Digital Object Identifier 10.1109/TIT.2010.2059720

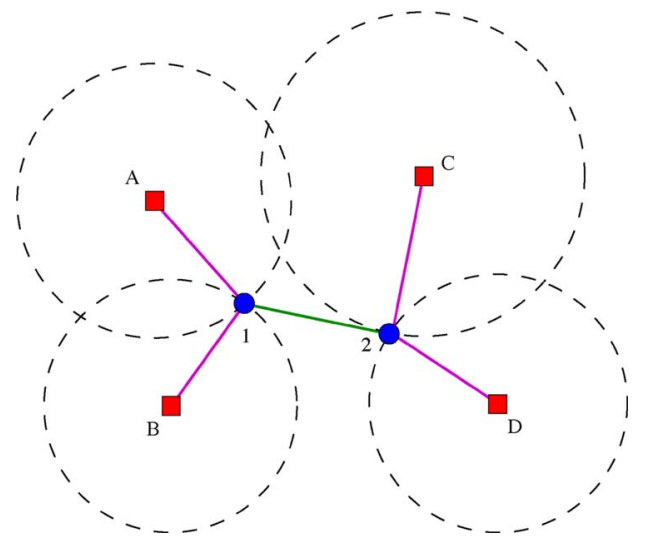

Fig. 1. Cooperative localization: the anchors (A, B, C, and D) communicate with the agents (1 and 2). Agent 1 is not in the communication/ranging range of anchor $\mathrm{C}$ and $\mathrm{D}$, while agent 2 is not in the communication/ranging range of anchors A and B. Neither agents can trilaterate its position based solely on the information from its neighboring anchors. However, cooperation between agents 1 and 2 enables both agents to be localized.

unknown positions. Conventionally, each agent localizes itself based on range measurements from at least three distinct anchors (in 2-D localization). Two common examples include the global positioning system (GPS) [9], [10] and beacon localization [11], [12]. In GPS, an agent can determine its location based on the signals received from a constellation of GPS satellites. However, GPS does not operate well in harsh environments, such as indoors or in urban canyons, since the signals cannot propagate through obstacles [7]-[9]. Beacon localization, on the other hand, relies on terrestrial anchors, such as WiFi access points or GSM base stations [11], [12]. However, in areas where network coverage is sparse, e.g., in emergency situations, localization errors can be unacceptably large.

Conventionally, high-accuracy localization can only be achieved using high-power anchors or a high-density anchor deployment, both of which are cost prohibitive and impractical in realistic settings. Hence, there is a need for localization systems that can achieve high accuracy in harsh environments with limited infrastructure requirements [6]-[8]. A practical way to address this need is through a combination of wideband transmission and cooperative localization. The fine delay resolution and robustness of wide bandwidth or ultrawide bandwidth (UWB) transmission enable accurate and reliable range (distance) measurements in harsh environments [13]-[18]. ${ }^{1}$ Hence, these transmission techniques are particularly well suited for localization. Cooperative localization is an emerging paradigm that circumvents the needs for high-power, high-density anchor deployment, and offers additional localization

${ }^{1}$ Other aspects of UWB technology can be found in [19]-[25]. 
accuracy by enabling the agents to help each other in estimating their positions [5], [6], [26]-[28]. In Fig. 1, for example, since each agent is in the communication/ranging range of only two anchors, neither agents can trilaterate its position based solely on the information from its neighboring anchors. However, cooperation enables both agents to be localized.

Understanding the fundamental limits of localization is crucial not only for providing a performance benchmark but also for guiding the deployment and operation of location-aware networks. Localization accuracy is fundamentally limited due to random phenomena such as noise, fading, shadowing, and multipath propagation. The impact of these phenomena has been investigated for noncooperative localization [7], [8], [29]-[31]. However, little is known regarding the bounds for cooperative localization. In particular, bounds on the cooperative localization performance were previously derived in [27] and [28] using only specific ranging models. In other words, these works start from signal metrics, extracted from the received waveforms. ${ }^{2}$ Such a process may discard information relevant for localization. Furthermore, the statistical models for those signal metrics depend heavily on the measurement processes. For instance, the ranging error of the time-of-arrival (TOA) metric is commonly modeled as additive Gaussian [27], [28], [31]. However, other studies (both theoretical [15], [38], [39] and experimental [8], [18]) indicate that the ranging error is not Gaussian. Hence, when deriving the fundamental limits of localization accuracy, it is important to start from the received waveforms rather than from signal metrics extracted from those waveforms.

In Part I [29], we have developed a general framework to characterize the localization accuracy of a given agent. In this paper, we build on the framework and determine fundamental properties of cooperative location-aware networks employing wideband transmission. The main contributions of this paper are as follows.

- We derive the fundamental limits of localization accuracy for wideband wireless cooperative networks in terms of a performance measure called the squared position error bound (SPEB).

- We employ the notion of equivalent Fisher information (EFI) to derive the network localization information, and show that this information can be decomposed into basic building blocks associated with every pair of the nodes, called the ranging information (RI).

- We quantify the contribution of the a priori knowledge of the channel parameters and the agents' positions to the network localization information, and show that agents and anchors can be treated in a unified way: anchors are special agents with infinite a priori position knowledge.

- We put forth a geometric interpretation of the EFI matrix (EFIM) using eigendecomposition, providing insights into the network localization problem.

- We derive scaling laws for the SPEB for both dense and extended location-aware networks, characterizing the behavior of cooperative location-aware networks in an asymptotic regime.

${ }^{2}$ Commonly used signal metrics include time-of-arrival (TOA) [7], [8], [15], [17], [32], time-difference-of-arrival (TDOA) [33], [34], angle-of-arrival (AOA) [7], [35], and received signal strength (RSS) [7], [36], [37].
The proposed framework generalizes the existing work on noncooperative localization [29] to cooperative networks, provides insights into the network localization problem, and can guide the design and deployment of location-aware networks.

The rest of the paper is organized as follows. Section II presents the system model and the concept of SPEB. In Section III, we apply the notion of EFI to derive the SPEB. Then, in Section IV, we provide a geometric interpretation of EFIM for localization and derive scaling laws for the SPEB. Finally, numerical results are given in Section $\mathrm{V}$, and conclusions are drawn in the last section.

Notation: The notation $\mathbb{E}_{\mathbf{x}}\{\cdot\}$ is the expectation operator with respect to the random vectors $\mathbf{x} ; \mathbf{A} \succ \mathbf{B}$ and $\mathbf{A} \succeq \mathbf{B}$ denote that the matrix $\mathbf{A}-\mathbf{B}$ is positive definite and positive semidefinite, respectively; $\operatorname{tr}\{\cdot\}$ denotes the trace of a square matrix; $[\cdot]^{\mathrm{T}}$ denotes the transpose of its argument; $[\cdot]_{n \times n, k}$ denotes the $k$ th $n \times n$ submatrix that starts from element $n(k-$ $1)+1$ on the diagonal of its argument; $[\cdot]_{r_{1}: r_{2}, c_{1}: c_{2}}$ denotes a submatrix composed of the rows $r_{1}$ to $r_{2}$ and the columns $c_{1}$ to $c_{2}$ of its argument; and $\|\cdot\|$ denotes the Euclidean norm of its argument. We also denote by $f(\mathbf{x})$ the probability density function (pdf) $f_{\mathbf{X}}(\mathbf{x})$ of the random vector $\mathbf{X}$ unless specified otherwise.

\section{SYSTEM MODEL}

In this section, we describe the wideband channel model and formulate the localization problem. We briefly review the information inequality and the performance measure called SPEB.

\section{A. Signal Model}

Consider a synchronous network consisting of $N_{\mathrm{b}}$ anchors (or beacons) and $N_{\mathrm{a}}$ agents with fixed topology. ${ }^{3}$ Anchors have perfect knowledge of their positions, while each agent attempts to estimate its position based on the waveforms received from neighboring nodes (see Fig. 1). Unlike conventional localization techniques, we consider a cooperative setting, where agents utilize waveforms received from neighboring agents in addition to those from anchors. The set of agents is denoted by $\mathcal{N}_{\mathrm{a}}=\left\{1,2, \ldots, N_{\mathrm{a}}\right\}$, while the set of anchors is $\mathcal{N}_{\mathrm{b}}=\left\{N_{\mathrm{a}}+\right.$ $\left.1, N_{\mathrm{a}}+2, \ldots, N_{\mathrm{a}}+N_{\mathrm{b}}\right\}$. The position of node $k$ is denoted by $\mathbf{p}_{k} \triangleq\left[\begin{array}{ll}x_{k} & y_{k}\end{array}\right]^{\mathrm{T}} .4$ Let $\phi_{k j}$ denote the angle from node $k$ to node $j$, i.e.,

$$
\phi_{k j}=\tan ^{-1} \frac{y_{k}-y_{j}}{x_{k}-x_{j}}
$$

and $\mathbf{q}_{k j} \triangleq\left[\begin{array}{ll}\cos \phi_{k j} & \sin \phi_{k j}\end{array}\right]^{\mathrm{T}}$ denote the corresponding unit vector.

The received waveform at the $k$ th agent $\left(k \in \mathcal{N}_{\mathrm{a}}\right)$ from the $j$ th node $\left(j \in \mathcal{N}_{\mathrm{b}} \cup \mathcal{N}_{\mathrm{a}} \backslash\{k\}\right)$ can be written as [24], [41]

$$
r_{k j}(t)=\sum_{l=1}^{L_{k j}} \alpha_{k j}^{(l)} s\left(t-\tau_{k j}^{(l)}\right)+z_{k j}(t), \quad t \in\left[0, T_{\mathrm{ob}}\right)
$$

${ }^{3}$ We consider synchronous networks for notional convenience. Our approach is also valid for asynchronous networks, where devices employ round-trip time-of-flight measurements [25], [40].

${ }^{4}$ For convenience, we focus on 2-D localization where $\mathbf{p}_{k} \in \mathbb{R}^{2}$, and we will later mention extensions to 3-D localization. 
where $s(t)$ is a known wideband waveform with Fourier transform $S(f), \alpha_{k j}^{(l)}$ and $\tau_{k j}^{(l)}$ are the amplitude and delay, respectively, of the $l$ th path, ${ }^{5} L_{k j}$ is the number of multipath components, $z_{k j}(t)$ represents the observation noise, modeled as additive white Gaussian processes with two-sided power spectral density $N_{0} / 2$, and $\left[0, T_{\mathrm{ob}}\right)$ is the observation interval. The relationship between the positions of nodes and the delays of the propagation paths is

$$
\tau_{k j}^{(l)}=\frac{1}{c}\left[\left\|\mathbf{p}_{k}-\mathbf{p}_{j}\right\|+b_{k j}^{(l)}\right], \quad j \in \mathcal{N}_{\mathrm{b}} \cup \mathcal{N}_{\mathrm{a}} \backslash\{k\}
$$

where $c$ is the propagation speed of the signal, and $b_{k j}^{(l)} \geq 0$ is a range bias induced by nonline-of-sight (NLOS) propagation. Line-of-sight (LOS) signals occur when the direct path between nodes $k$ and $j$ is unobstructed, such that $b_{k j}^{(1)}=0$.

\section{B. Error Bounds on Position Estimation}

We first introduce $\boldsymbol{\theta}$ as the vector of unknown parameters

$$
\boldsymbol{\theta}=\left[\begin{array}{lllll}
\mathbf{P}^{\mathrm{T}} & \tilde{\boldsymbol{\theta}}_{1}^{\mathrm{T}} & \tilde{\boldsymbol{\theta}}_{2}^{\mathrm{T}} & \cdots & \tilde{\boldsymbol{\theta}}_{N_{\mathrm{a}}^{\mathrm{a}}}^{\mathrm{T}}
\end{array}\right]^{\mathrm{T}}
$$

where $\mathbf{P}$ consists of all the agents' positions

$$
\mathbf{P}=\left[\begin{array}{llll}
\mathbf{p}_{1}^{\mathrm{T}} & \mathbf{p}_{2}^{\mathrm{T}} & \cdots & \mathbf{p}_{N_{\mathrm{a}}}^{\mathrm{T}}
\end{array}\right]^{\mathrm{T}}
$$

and $\tilde{\boldsymbol{\theta}}_{k}$ is the vector of the multipath parameters associated with the waveforms received at the $k$ th agent ${ }^{6}$

$$
\tilde{\boldsymbol{\theta}}_{k}=\left[\begin{array}{llllll}
\boldsymbol{\kappa}_{k, 1}^{\mathrm{T}} & \cdots & \boldsymbol{\kappa}_{k, k-1}^{\mathrm{T}} & \boldsymbol{\kappa}_{k, k+1}^{\mathrm{T}} & \cdots & \boldsymbol{\kappa}_{k, N_{\mathrm{a}}+N_{\mathrm{b}}}^{\mathrm{T}}
\end{array}\right]^{\mathrm{T}}
$$

in which $\kappa_{k j}$ is the vector of the multipath parameters associated with $r_{k j}(t)^{7}$

$$
\boldsymbol{\kappa}_{k j}=\left[\begin{array}{lllll}
b_{k j}^{(1)} & \alpha_{k j}^{(1)} & \cdots & b_{k j}^{\left(L_{k j}\right)} & \alpha_{k j}^{\left(L_{k j}\right)}
\end{array}\right]^{\mathrm{T}} .
$$

Second, we introduce $\mathbf{r}$ as the vector representation of all the received waveforms, given by $\mathbf{r}=\left[\begin{array}{llll}\mathbf{r}_{1}^{\mathrm{T}} & \mathbf{r}_{2}^{\mathrm{T}} & \cdots & \mathbf{r}_{N_{\mathrm{a}}}^{\mathrm{T}}\end{array}\right]^{\mathrm{T}}$, where

$$
\mathbf{r}_{k}=\left[\begin{array}{llllll}
\mathbf{r}_{k, 1}^{\mathrm{T}} & \cdots & \mathbf{r}_{k, k-1}^{\mathrm{T}} & \mathbf{r}_{k, k+1}^{\mathrm{T}} & \cdots & \mathbf{r}_{k, N_{\mathrm{a}}+N_{\mathrm{b}}}^{\mathrm{T}}
\end{array}\right]^{\mathrm{T}}
$$

in which $\mathbf{r}_{k j}$ is obtained from the Karhunen-Loève (KL) expansion of $r_{k j}(t)$ [42], [43]. We tacitly assume that when nodes $j$ and $k$ cannot communicate directly, the corresponding entry $\mathbf{r}_{k j}$ is omitted in $\mathbf{r}$.

We can now introduce an estimator $\hat{\boldsymbol{\theta}}$ of the unknown parameter $\boldsymbol{\theta}$ based on the observation $\mathbf{r}$. The mean squared error (MSE) matrix of $\hat{\boldsymbol{\theta}}$ satisfies the information inequality [42]-[44]

$$
\mathbb{E}_{\mathbf{r}, \boldsymbol{\theta}}\left\{(\hat{\boldsymbol{\theta}}-\boldsymbol{\theta})(\hat{\boldsymbol{\theta}}-\boldsymbol{\theta})^{\mathrm{T}}\right\} \succeq \mathbf{J}_{\boldsymbol{\theta}}^{-1}
$$

${ }^{5} \mathrm{We}$ consider the general case where the wideband channel is not necessarily reciprocal. Our results can be easily specialized to the reciprocal case, where we have $L_{k j}=L_{j k}, \alpha_{k j}^{(l)}=\alpha_{j k}^{(l)}$, and $\tau_{k j}^{(l)}=\tau_{j k}^{(l)}$ hence $b_{k j}^{(l)}=b_{j k}^{(l)}$, for $l=1,2, \ldots, L_{k j}$.

${ }^{6}$ In cases where the channel is reciprocal, only half of the multipath parameters are needed. Without loss of generality, we only use $\left\{\alpha_{k j}^{(l)}, b_{k j}^{(l)}: k, j \in\right.$ $\left.\mathcal{N}_{\mathrm{a}}, k>j\right\}$.

${ }^{7}$ The bias $b_{k j}^{(1)}=0$ for LOS signals. From the perspective of Bayesian estimation, it can be thought of as a random parameter with infinite a priori Fisher information [29]. where $\mathbf{J}_{\boldsymbol{\theta}}$ is the Fisher information matrix (FIM) for $\boldsymbol{\theta},{ }^{8}$ given by

$$
\mathbf{J}_{\boldsymbol{\theta}}=\mathbb{E}_{\mathbf{r}, \boldsymbol{\theta}}\left\{-\frac{\partial^{2}}{\partial \boldsymbol{\theta} \partial \boldsymbol{\theta}^{\mathrm{T}}} \ln f(\mathbf{r}, \boldsymbol{\theta})\right\}
$$

in which $f(\mathbf{r}, \boldsymbol{\theta})$ is the joint pdf of the observation $\mathbf{r}$ and the parameter vector $\boldsymbol{\theta}$. For an estimate $\hat{\mathbf{p}}_{k}$ of the $k$ th agent's position, (3) implies that

$$
\mathbb{E}_{\mathbf{r}, \boldsymbol{\theta}}\left\{\left(\hat{\mathbf{p}}_{k}-\mathbf{p}_{k}\right)\left(\hat{\mathbf{p}}_{k}-\mathbf{p}_{k}\right)^{\mathrm{T}}\right\} \succeq\left[\mathbf{J}_{\boldsymbol{\theta}}^{-1}\right]_{2 \times 2, k} .
$$

One natural measure for position accuracy is the average squared position error $\mathbb{E}_{\mathbf{r}, \boldsymbol{\theta}}\left\{\left\|\hat{\mathbf{p}}_{k}-\mathbf{p}_{k}\right\|^{2}\right\}$, which can be bounded below by $\mathcal{P}\left(\mathbf{p}_{k}\right)$ defined in the following.

Definition 1 (SPEB [29]): The SPEB of the $k$ th agent is defined to be

$$
\mathcal{P}\left(\mathbf{p}_{k}\right) \triangleq \operatorname{tr}\left\{\left[\mathbf{J}_{\boldsymbol{\theta}}^{-1}\right]_{2 \times 2, k}\right\} .
$$

Since the error of the position estimate $\hat{\mathbf{p}}_{k}-\mathbf{p}_{k}$ is a vector, it may also be of interest to know the position error in a particular direction. The directional position error along a given unit vector $\mathbf{u}$ is the position error projected on it, i.e., $\mathbf{u}^{\mathrm{T}}\left(\hat{\mathbf{p}}_{k}-\mathbf{p}_{k}\right)$, and its average squared error $\mathbb{E}_{\mathbf{r}, \boldsymbol{\theta}}\left\{\left\|\mathbf{u}^{\mathrm{T}}\left(\hat{\mathbf{p}}_{k}-\mathbf{p}_{k}\right)\right\|^{2}\right\}$ can be bounded below by $\mathcal{P}\left(\mathbf{p}_{k} ; \mathbf{u}\right)$ defined in the following. ${ }^{9}$

Definition 2 (Directional Position Error Bound): The directional position error bound (DPEB) of the $k$ th agent with constraint $\mathbf{u}_{\perp}^{\mathrm{T}}\left(\hat{\mathbf{p}}_{k}-\mathbf{p}_{k}\right)=0$ is defined to be

$$
\mathcal{P}\left(\mathbf{p}_{k} ; \mathbf{u}\right) \triangleq \mathbf{u}^{\mathrm{T}}\left[\mathbf{J}_{\boldsymbol{\theta}}^{-1}\right]_{2 \times 2, k} \mathbf{u}
$$

where $\mathbf{u}, \mathbf{u}_{\perp} \in \mathbb{R}^{2}$ are unit vectors such that $\left\langle\mathbf{u}, \mathbf{u}_{\perp}\right\rangle=0$.

Proposition 1: The SPEB of the $k$ th agent is the sum of the DPEBs in any two orthogonal directions, i.e.,

$$
\mathcal{P}\left(\mathbf{p}_{k}\right)=\mathcal{P}\left(\mathbf{p}_{k} ; \mathbf{u}\right)+\mathcal{P}\left(\mathbf{p}_{k} ; \mathbf{u}_{\perp}\right) .
$$

Proof: See Appendix I.

\section{Joint PDF of Observations and Parameters}

Evaluation of (4) requires knowledge of the joint distribution $f(\mathbf{r}, \boldsymbol{\theta})$. We can write $f(\mathbf{r}, \boldsymbol{\theta})=f(\mathbf{r} \mid \boldsymbol{\theta}) f(\boldsymbol{\theta})$, where $f(\mathbf{r} \mid \boldsymbol{\theta})$ is the likelihood function, and $f(\boldsymbol{\theta})$ is the a priori distribution of the parameter $\boldsymbol{\theta} \cdot{ }^{10}$ In this section, we describe the structure of both functions in detail.

Since the received waveforms $r_{k j}(t)$ are independent conditioned on the parameter $\boldsymbol{\theta}, f(\mathbf{r} \mid \boldsymbol{\theta})$ can be expressed as [42], [43]

$$
f(\mathbf{r} \mid \boldsymbol{\theta})=\prod_{k \in \mathcal{N}_{\mathrm{a}}} \prod_{j \in \mathcal{N}_{\mathrm{b}} \cup \mathcal{N}_{\mathrm{a}} \backslash\{k\}} f\left(\mathbf{r}_{k j} \mid \boldsymbol{\theta}\right)
$$

${ }^{8}$ With a slight abuse of notation, $\mathbb{E}_{\mathbf{r}, \boldsymbol{\theta}}\{\cdot\}$ in (3) and (4) will be used for deterministic, random, and hybrid cases, with the understanding that the expectation operation is not performed over the deterministic components of $\boldsymbol{\theta}$ [43], [44]. Note also that for the deterministic components, the lower bound is valid for their unbiased estimates.

${ }^{9}$ In higher dimensions, this notion can be extend to the position error in any subspaces, such as a hyperplane.

${ }^{10}$ When a subset of the parameters are deterministic, they are eliminated from $f(\boldsymbol{\theta})$. 
where

$$
\begin{aligned}
f\left(\mathbf{r}_{k j} \mid \boldsymbol{\theta}\right) \propto & \exp \left\{\frac{2}{N_{0}} \int_{0}^{T_{\mathrm{ob}}} r_{k j}(t) \sum_{l=1}^{L_{k j}} \alpha_{k j}^{(l)} s\left(t-\tau_{k j}^{(l)}\right) d t\right. \\
& \left.-\frac{1}{N_{0}} \int_{0}^{T_{\mathrm{ob}}}\left[\sum_{l=1}^{L_{k j}} \alpha_{k j}^{(l)} s\left(t-\tau_{k j}^{(l)}\right)\right]^{2} d t\right\} .
\end{aligned}
$$

When the multipath parameters $\boldsymbol{\kappa}_{k j}$ are independent conditioned on the nodes' positions, ${ }^{11} f(\boldsymbol{\theta})$ can be expressed as

$$
\begin{aligned}
f(\boldsymbol{\theta}) & =f(\mathbf{P}) \prod_{k \in \mathcal{N}_{\mathrm{a}}} f\left(\tilde{\boldsymbol{\theta}}_{k} \mid \mathbf{P}\right) \\
& =f(\mathbf{P}) \prod_{k \in \mathcal{N}_{\mathrm{a}}} \prod_{j \in \mathcal{N}_{\mathrm{b}} \cup \mathcal{N}_{\mathrm{a}} \backslash\{k\}} f\left(\boldsymbol{\kappa}_{k j} \mid \mathbf{P}\right)
\end{aligned}
$$

where $f(\mathbf{P})$ is the joint pdf of all the agents' positions, and $f\left(\boldsymbol{\kappa}_{k j} \mid \mathbf{P}\right)$ is the joint pdf of the multipath parameters $\boldsymbol{\kappa}_{k j}$ conditioned on the agents' positions. Based on existing propagation models for wideband and UWB channels [14], [25], the joint pdf of the channel parameters can be further written as [29]

$$
f\left(\boldsymbol{\kappa}_{k j} \mid \mathbf{P}\right)=f\left(\boldsymbol{\kappa}_{k j} \mid d_{k j}\right)
$$

where $d_{k j}=\left\|\mathbf{p}_{k}-\mathbf{p}_{j}\right\|$ for $k \in \mathcal{N}_{\mathrm{a}}$ and $j \in \mathcal{N}_{\mathrm{b}} \cup \mathcal{N}_{\mathrm{a}} \backslash\{k\}$.

Combining (8) and (6) leads to

$$
\begin{aligned}
\ln f(\mathbf{r}, \boldsymbol{\theta})= & \sum_{k \in \mathcal{N}_{\mathrm{a}}} \sum_{j \in \mathcal{N}_{\mathrm{b}}}\left[\ln f\left(\mathbf{r}_{k j} \mid \boldsymbol{\theta}\right)+\ln f\left(\boldsymbol{\kappa}_{k j} \mid \mathbf{P}\right)\right] \\
& +\sum_{k \in \mathcal{N}_{\mathrm{a}}} \sum_{j \in \mathcal{N}_{\mathrm{a}} \backslash\{k\}}\left[\ln f\left(\mathbf{r}_{k j} \mid \boldsymbol{\theta}\right)+\ln f\left(\boldsymbol{\kappa}_{k j} \mid \mathbf{P}\right)\right] \\
& +\ln f(\mathbf{P})
\end{aligned}
$$

where the first and second groups of summation account for the information from anchors and that from agents' cooperation, respectively, and the last term accounts for the information from the a priori knowledge of the agents' positions. This implies that the FIM for $\boldsymbol{\theta}$ in (4) can be written as $\mathbf{J}_{\boldsymbol{\theta}}=\mathbf{J}_{\boldsymbol{\theta}}^{\mathrm{A}}+\mathbf{J}_{\boldsymbol{\theta}}^{\mathrm{C}}+\mathbf{J}_{\boldsymbol{\theta}}^{\mathrm{P}}$, where $\mathbf{J}_{\boldsymbol{\theta}}^{\mathrm{A}}, \mathbf{J}_{\boldsymbol{\theta}}^{\mathrm{C}}$, and $\mathbf{J}_{\boldsymbol{\theta}}^{\mathrm{P}}$ correspond to the localization information from anchors, agents' cooperation, and a priori knowledge of the agents' positions, respectively.

\section{EVALUATION OF FIM}

In this section, we briefly review the notion of EFI [29] and apply it to derive the SPEB for each agent. We consider both the cases with and without a priori knowledge of the agents' positions. We also introduce the concept of RI, which turns out to be the basic building block for the EFIM.

\section{A. EFIM and RI}

We saw in the previous section that the SPEB can be obtained by inverting the FIM $\mathbf{J}_{\boldsymbol{\theta}}$ in (4). However, $\mathbf{J}_{\boldsymbol{\theta}}$ is a matrix of very high dimensions, while only a much smaller submatrix $\left[\mathbf{J}_{\boldsymbol{\theta}}^{-1}\right]_{2 N_{\mathrm{a}} \times 2 N_{\mathrm{a}}}$ is of interest. To gain insights into localization

\footnotetext{
${ }^{11}$ This is a common model for analyzing wideband communication, unless two nodes are close to each other so that the channels from a third node to them are correlated. Our analysis can also account for the correlated channels, in which case the SPEB will be higher than that corresponding to the independent channels.
}

problem, we will employ the notions of EFIM and RI [29]. For the completeness of the paper, we briefly review the notions in the following.

Definition 3 (EFIM): Given a parameter vector $\boldsymbol{\theta}=\left[\begin{array}{ll}\boldsymbol{\theta}_{1}^{\mathrm{T}} & \boldsymbol{\theta}_{2}^{\mathrm{T}}\end{array}\right]^{\mathrm{T}}$ and the FIM $\mathbf{J}_{\boldsymbol{\theta}}$ of the form

$$
\mathbf{J}_{\boldsymbol{\theta}}=\left[\begin{array}{cc}
\mathbf{A} & \mathbf{B} \\
\mathbf{B}^{\mathrm{T}} & \mathbf{C}
\end{array}\right]
$$

where $\boldsymbol{\theta} \in \mathbb{R}^{N}, \boldsymbol{\theta}_{1} \in \mathbb{R}^{n}, \mathbf{A} \in \mathbb{R}^{n \times n}, \mathbf{B} \in \mathbb{R}^{n \times(N-n)}$, and $\mathbf{C} \in \mathbb{R}^{(N-n) \times(N-n)}$ with $n<N$, the EFIM for $\boldsymbol{\theta}_{1}$ is given by

$$
\mathbf{J}_{\mathrm{e}}\left(\boldsymbol{\theta}_{1}\right) \triangleq \mathbf{A}-\mathbf{B} \mathbf{C}^{-1} \mathbf{B}^{\mathrm{T}}
$$

Note that the EFIM retains all the necessary information to derive the information inequality for the parameter $\boldsymbol{\theta}_{1}$, in a sense that $\left[\mathbf{J}_{\boldsymbol{\theta}}^{-1}\right]_{n \times n}=\left[\mathbf{J}_{\mathrm{e}}\left(\boldsymbol{\theta}_{1}\right)\right]^{-1}$, so that the MSE matrix of the estimates of $\boldsymbol{\theta}_{1}$ is "bounded" below by $\left[\mathbf{J}_{\mathrm{e}}\left(\boldsymbol{\theta}_{1}\right)\right]^{-1}$. The righthand side of (11) is known as the Schur's complement of matrix A [45], and it has been used for simplifying the Cramér-Rao bounds (CRBs) [31], [32], [46].

Definition $4(R I)$ : The RI is a $2 \times 2$ matrix of the form $\lambda \mathbf{J}_{\mathrm{r}}(\phi)$, where $\lambda$ is a nonnegative number called the ranging information intensity (RII) and the matrix $\mathbf{J}_{\mathbf{r}}(\phi)$ is called the ranging direction matrix (RDM) with the following structure:

$$
\mathbf{J}_{\mathbf{r}}(\phi) \triangleq\left[\begin{array}{cc}
\cos ^{2} \phi & \cos \phi \sin \phi \\
\cos \phi \sin \phi & \sin ^{2} \phi
\end{array}\right] \text {. }
$$

The RDM $\mathbf{J}_{\mathrm{r}}(\phi)$ has exactly one nonzero eigenvalue equal to 1 with corresponding eigenvector $\mathbf{q}=\left[\begin{array}{ll}\cos \phi & \sin \phi\end{array}\right]^{\mathrm{T}}$, i.e., $\mathbf{J}_{\mathbf{r}}(\phi)=\mathbf{q} \mathbf{q}^{\mathrm{T}}$. Thus, the corresponding RI is " $1-\mathrm{D}$ " along the direction $\phi$.

\section{B. EFIM Without a Priori Position Knowledge}

In this section, we consider the case in which a priori knowledge of the agents' positions is unavailable, i.e., $f(\mathbf{P})$ is eliminated from (8). We first prove a general theorem, describing the structure of the EFIM, followed by a special case, where there is no a priori knowledge regarding the channel parameters.

Theorem 1: When a priori knowledge of the agents' positions is unavailable, and the channel parameters corresponding to different waveforms are mutually independent, the EFIM for the agents' positions is a $2 N_{\mathrm{a}} \times 2 N_{\mathrm{a}}$ matrix, structured as (12), shown at the bottom of the next page, where $\mathbf{J}_{\mathrm{e}}^{\mathrm{A}}\left(\mathbf{p}_{k}\right)$ and $\mathbf{C}_{k j}$ can be expressed in terms of the RI

$$
\mathbf{J}_{\mathrm{e}}^{\mathrm{A}}\left(\mathbf{p}_{k}\right)=\sum_{j \in \mathcal{N}_{\mathrm{b}}} \lambda_{k j} \mathbf{J}_{\mathrm{r}}\left(\phi_{k j}\right)
$$

and

$$
\mathbf{C}_{k j}=\mathbf{C}_{j k}=\left(\lambda_{k j}+\lambda_{j k}\right) \mathbf{J}_{\mathrm{r}}\left(\phi_{k j}\right)
$$

with $\lambda_{k j}$ given by (35) in Appendix II.

Proof: See Appendix II.

Remark 1: We make the following remarks.

- To obtain the SPEB of a specific agent, we can apply EFI analysis again and further reduce $\mathbf{J}_{\mathrm{e}}(\mathbf{P})$ into a $2 \times 2$ EFIM. 
- The RI is the basic building block of the EFIM for localization, and each RI corresponds to an individual received waveform. The RII $\lambda_{k j}$ is determined by the power and bandwidth of the received waveform, the multipath propagation, as well as the a priori channel knowledge. Note that each received waveform provides only 1-D information for localization along the angle $\phi_{k j}$.

- The EFIM $\mathbf{J}_{\mathrm{e}}(\mathbf{P})$ can be decomposed into localization information from anchors and that from agents' cooperation. The former part is represented as a block-diagonal matrix whose nonzero elements are $\mathbf{J}_{\mathrm{e}}^{\mathrm{A}}\left(\mathbf{p}_{k}\right)$, for the $k$ th agent, and each $\mathbf{J}_{\mathrm{e}}^{\mathrm{A}}\left(\mathbf{p}_{k}\right)$ is a weighted sum of RDMs over anchors. Hence, the localization information from anchors is not interrelated among agents. The latter part is a highly structured matrix consisting of RIs $\mathbf{C}_{k j}$. Hence, the localization information from agents' cooperation is highly interrelated. This is intuitive since the effectiveness of the localization information provided by a particular agent depends on its position error.

Theorem 2: When a priori knowledge of the agents' positions and the channel parameters is unavailable, the EFIM for the agents' positions is a $2 N_{\mathrm{a}} \times 2 N_{\mathrm{a}}$ matrix, structured as in (12) with the RII $\lambda_{k j}$ given by

$$
\lambda_{k j}= \begin{cases}8 \pi^{2} \beta^{2} / c^{2} \cdot\left(1-\chi_{k j}\right) \mathrm{SNR}_{k j}^{(1)}, & \text { LOS signal } \\ 0, & \text { NLOS signal }\end{cases}
$$

where $\beta$ is the effective bandwidth of transmitted waveform $s(t)$

$$
\beta=\left(\frac{\int_{-\infty}^{+\infty} f^{2}|S(f)|^{2} d f}{\int_{-\infty}^{+\infty}|S(f)|^{2} d f}\right)^{1 / 2}
$$

$\mathrm{SNR}_{k j}^{(1)}$ is the SNR of the first path in $r_{k j}(t)$

$$
\mathrm{SNR}_{k j}^{(1)}=\frac{\left|\alpha_{k j}^{(1)}\right|^{2} \int_{-\infty}^{+\infty}|S(f)|^{2} d f}{N_{0}}
$$

and $0 \leq \chi_{k j} \leq 1$ is called the path-overlap coefficient, which depends on the first contiguous cluster ${ }^{12}$ in LOS signals.

Proof: See Appendix III.

Remark 2: We make the following remarks.

- The theorem shows that when a priori knowledge of channel parameters is unavailable, the NLOS signals do not contribute to localization accuracy, and hence these signals can be discarded. This agrees with the previous

\footnotetext{
${ }^{12}$ The first contiguous cluster is the first group of nondisjoint paths. Two paths that arrive at time $\tau_{i}$ and $\tau_{j}$ are called nondisjoint if $\left|\tau_{i}-\tau_{j}\right|$ is less than the duration of $s(t)$ [29].
}

observations in [8], [31], and [32] although the authors considered different models.

- For LOS signals, the RII is determined by the first contiguous cluster [29], implying that it is not necessary to process the latter multipath components. In particular, the RII is determined by the effective bandwidth $\beta$, the first path's SNR, and the propagation effect characterized by $\chi_{k j}$.

- Since $\chi_{k j} \geq 0$, path overlap always deteriorates the accuracy unless $\chi_{k j}=0$, in which the first signal component $s\left(t-\tau_{k j}^{(1)}\right)$ does not overlap with later components $s\left(t-\tau_{k j}^{(l)}\right)$ for $l>1$.

\section{EFIM With a Priori Position Knowledge}

We now consider the case in which the a priori knowledge of the agents' positions, characterized by $f(\mathbf{P})$, is available. We first derive the EFIM, based on which we prove that agents and anchors can be treated in a unified way under this framework. We then present a special scenario in which the a priori knowledge of the agents' positions satisfies certain conditions so that we can gain insights into the EFIM.

Theorem 3: When a priori knowledge of the agents' positions is available, and the channel parameters corresponding to different waveforms are mutually independent, the EFIM for the agents' positions is a $2 N_{\mathrm{a}} \times 2 N_{\mathrm{a}}$ matrix, given by ${ }^{13}$

$$
\mathbf{J}_{\mathrm{e}}(\mathbf{P})=\mathbf{J}_{\mathrm{e}}^{\mathrm{A}}(\mathbf{P})+\mathbf{J}_{\mathrm{e}}^{\mathrm{C}}(\mathbf{P})+\boldsymbol{\Xi}_{\mathbf{P}}
$$

where

$$
\begin{aligned}
& {\left[\mathbf{J}_{\mathbf{e}}^{\mathrm{A}}(\mathbf{P})\right]_{2 k-1: 2 k, 2 m-1: 2 m}} \\
& = \begin{cases}\sum_{j \in \mathcal{N}_{\mathrm{b}}} \mathbf{R}_{k}\left(\mathbf{r}_{k j}\right), & k=m \\
\mathbf{0}, & k \neq m\end{cases} \\
& {\left[\mathbf{J}_{\mathrm{e}}^{\mathrm{C}}(\mathbf{P})\right]_{2 k-1: 2 k, 2 m-1: 2 m}} \\
& = \begin{cases}\sum_{j \in \mathcal{N}_{a} \backslash\{k\}}\left[\mathbf{R}_{k}\left(\mathbf{r}_{k j}\right)+\mathbf{R}_{k}\left(\mathbf{r}_{j k}\right)\right], & k=m \\
-\left[\mathbf{R}_{k}\left(\mathbf{r}_{k m}\right)+\mathbf{R}_{k}\left(\mathbf{r}_{m k}\right)\right], & k \neq m\end{cases}
\end{aligned}
$$

and

$$
\boldsymbol{\Xi}_{\mathbf{P}}=\mathbb{E}_{\mathbf{P}}\left\{-\frac{\partial^{2}}{\partial \mathbf{P} \partial \mathbf{P}^{\mathrm{T}}} \ln f(\mathbf{P})\right\}
$$

with $\mathbf{R}_{k}\left(\mathbf{r}_{k j}\right) \in \mathbb{R}^{2 \times 2}$ given by (15), shown at the bottom of the next page. Block matrix $\boldsymbol{\Phi}_{k j}(\mathbf{x}, \mathbf{y})$ in (15) is defined as (27) in Appendix II.

Proof: See Appendix IV.

${ }^{13}$ Note that $\mathbf{J}_{\mathrm{e}}(\mathbf{P})$ in (14) does not depend on any particular value of the random vector $\mathbf{P}$, whereas $\mathbf{J}_{\mathrm{e}}(\mathbf{P})$ in (12) is a function of the deterministic vector $\mathbf{P}$.

$$
\mathbf{J}_{\mathrm{e}}(\mathbf{P})=\left[\begin{array}{cccc}
\mathbf{J}_{\mathrm{e}}^{\mathrm{A}}\left(\mathbf{p}_{1}\right)+\sum_{j \in \mathcal{N}_{a} \backslash\{1\}} \mathbf{C}_{1, j} & -\mathbf{C}_{1,2} & \cdots & -\mathbf{C}_{1, N_{\mathrm{a}}} \\
-\mathbf{C}_{1,2} & \mathbf{J}_{\mathrm{e}}^{\mathrm{A}}\left(\mathbf{p}_{2}\right)+\sum_{j \in \mathcal{N}_{\mathrm{a}} \backslash\{2\}} \mathbf{C}_{2, j} & & -\mathbf{C}_{2, N_{\mathrm{a}}} \\
\vdots & & \ddots & \\
-\mathbf{C}_{1, N_{\mathrm{a}}} & -\mathbf{C}_{2, N_{\mathrm{a}}} & & \mathbf{J}_{\mathrm{e}}^{\mathrm{A}}\left(\mathbf{p}_{N_{\mathrm{a}}}\right)+\sum_{j \in \mathcal{N}_{\mathrm{a}} \backslash\left\{N_{\mathrm{a}}\right\}} \mathbf{C}_{N_{\mathrm{a}}, j}
\end{array}\right]
$$


Remark 3: The EFIM for agents' positions is derived in (14) for the case when a priori knowledge of the agents' positions is available. Compared to (12) in the Theorem 1, the EFIM in (14) retains the same structure of the localization information from both anchors and cooperation, except that all RIs in Theorem 3 are obtained by averaging the $2 \times 2$ matrices over the possible agents' positions. In addition, the localization information from the position knowledge is characterized in terms of an additive component $\boldsymbol{\Xi}_{\mathbf{P}}$. This knowledge improves localization because $\boldsymbol{\Xi}_{\mathbf{P}}$ is positive semidefinite.

Based on the result of Theorem 3, we can now treat anchors and agents in a unified way, as will be shown in the following theorem.

Theorem 4: Anchors are equivalent to agents with infinite a priori position knowledge in the following sense: when the $k$ th agent has infinite a priori position knowledge, i.e., $\boldsymbol{\Xi}_{\mathrm{p}_{k}}=$ $\lim _{t^{2} \rightarrow \infty} \operatorname{diag}\left\{t^{2}, t^{2}\right\}$, then

$$
\mathbf{J}_{\mathrm{e}}\left(\mathbf{P}_{\bar{k}}\right)=\left[\mathbf{J}_{\mathrm{e}}(\mathbf{P})\right]_{\bar{k}}
$$

where $\mathbf{P}_{\bar{k}}$ is the vector $\mathbf{P}$ without rows $2 k-1$ to $2 k$, and $\left[\mathbf{J}_{\mathrm{e}}(\mathbf{P})\right]_{k}$ is the matrix $\mathbf{J}_{\mathrm{e}}(\mathbf{P})$ without rows $2 k-1$ to $2 k$ and columns $2 k-1$ to $2 k$.

Proof: See Appendix V.

Remark 4: The theorem shows mathematically that agents are equivalent to anchors if they have infinite a priori position knowledge, which agrees with our intuition. As such, it is not necessary to distinguish between agents and anchors. This view will facilitate the analysis of location-aware networks and the design of localization algorithms: every agent can treat the information coming from anchors and other cooperating agents in a unified way.

The general expression of the EFIM for the case with a priori position knowledge is given in (14), which is much more involved than that for the case without position knowledge in (12). However, in the special case when

$$
\mathbb{E}_{\mathbf{P}}\{g(\mathbf{P})\}=g\left(\mathbb{E}_{\mathbf{P}}\{\mathbf{P}\}\right)
$$

for the functions $g(\cdot)$ involved in the derivation of the EFIM (see Appendix IV), ${ }^{14}$ we can gain insight into the structure of the EFIM as shown by the following corollary.

\footnotetext{
${ }^{14}$ This occurs when every agent's a priori position distribution is concentrated in a small area relative to the distance between the agent and the other nodes, so that $g(\mathbf{P})$ is flat in that area.
}

Corollary 1: When the a priori distribution of the agents' positions satisfies (16), and the channel parameters corresponding to different waveforms are mutually independent, the EFIM for the agents' positions is a $2 N_{\mathrm{a}} \times 2 N_{\mathrm{a}}$ matrix, structured as (17), shown at the bottom of the page, where $\overline{\mathbf{J}}_{\mathrm{e}}^{\mathrm{A}}\left(\mathbf{p}_{k}\right)$ and $\overline{\mathbf{C}}_{k j}$ can be expressed in terms of the RI

$$
\overline{\mathbf{J}}_{\mathrm{e}}^{\mathrm{A}}\left(\mathbf{p}_{k}\right)=\sum_{j \in \mathcal{N}_{\mathrm{b}}} \bar{\lambda}_{k j} \mathbf{J}_{\mathrm{r}}\left(\bar{\phi}_{k j}\right)
$$

and

$$
\overline{\mathbf{C}}_{k j}=\overline{\mathbf{C}}_{j k}=\left(\bar{\lambda}_{k j}+\bar{\lambda}_{j k}\right) \mathbf{J}_{\mathrm{r}}\left(\bar{\phi}_{k j}\right)
$$

where $\overline{\mathbf{P}}=\mathbb{E}_{\mathbf{P}}\{\mathbf{P}\}, \bar{\lambda}_{k j}$ is the RII given in (35) evaluated at $\overline{\mathbf{P}}$, and $\bar{\phi}_{k j}$ is the angle from $\overline{\mathbf{p}}_{k}$ to $\overline{\mathbf{p}}_{j}$.

Proof: See Appendix IV.

\section{Discussions}

We will now discuss the results derived in the previous sections. Our discussion includes 1) the EFIM for the agents in noncooperative localization, 2) an application of the cooperative localization to tracking, 3) a recursive method to construct an EFIM for large networks, and 4) the extension to 3-D scenarios.

1) Noncooperative Localization: When the agents do not cooperate, the matrices corresponding to the agents' cooperation in (12) in Theorem 1 and (17) in Corollary 1 are discarded. In particular, the EFIM $\mathbf{J}_{\mathrm{e}}(\mathbf{P})$ in Theorem 1 reverts to

$$
\mathbf{J}_{\mathrm{e}}(\mathbf{P})=\operatorname{diag}\left\{\mathbf{J}_{\mathrm{e}}^{\mathrm{A}}\left(\mathbf{p}_{1}\right), \mathbf{J}_{\mathrm{e}}^{\mathrm{A}}\left(\mathbf{p}_{2}\right), \ldots, \mathbf{J}_{\mathrm{e}}^{\mathrm{A}}\left(\mathbf{p}_{N_{\mathrm{a}}}\right)\right\}
$$

and hence the $2 \times 2$ EFIM for the $k$ th agent is equal to $\mathbf{J}_{\mathrm{e}}\left(\mathbf{p}_{k}\right)=$ $\mathbf{J}_{\mathrm{e}}^{\mathrm{A}}\left(\mathbf{p}_{k}\right)$. Similarly, the EFIM $\mathbf{J}_{\mathrm{e}}(\mathbf{P})$ in Corollary 1 reverts to

$$
\mathbf{J}_{\mathrm{e}}(\mathbf{P})=\operatorname{diag}\left\{\overline{\mathbf{J}}_{\mathrm{e}}^{\mathrm{A}}\left(\mathbf{p}_{1}\right), \overline{\mathbf{J}}_{\mathrm{e}}^{\mathrm{A}}\left(\mathbf{p}_{2}\right), \ldots, \overline{\mathbf{J}}_{\mathrm{e}}^{\mathrm{A}}\left(\mathbf{p}_{N_{\mathrm{a}}}\right)\right\}+\boldsymbol{\Xi}_{\mathbf{P}} .
$$

Furthermore, when the agents' positions are independent $a$ priori, $\boldsymbol{\Xi}_{\mathbf{P}}=\operatorname{diag}\left\{\boldsymbol{\Xi}_{\mathbf{p}_{1}}, \boldsymbol{\Xi}_{\mathbf{p}_{2}}, \ldots, \boldsymbol{\Xi}_{\mathbf{p}_{N_{\mathrm{a}}}}\right\}$ and the $2 \times 2$ EFIM for the $k$ th agent can be written as $\mathbf{J}_{\mathrm{e}}\left(\mathbf{p}_{k}\right)=\overline{\mathbf{J}}_{\mathrm{e}}^{\mathrm{A}}\left(\mathbf{p}_{k}\right)+\boldsymbol{\Xi}_{\mathbf{p}_{k}}$.

2) Spatial Versus Temporal Cooperation for Localization: Rather than multiple agents in cooperation, a single agent can "cooperate" with itself over time. Such temporal cooperative localization can easily be analyzed within our framework, as follows.

Consider a single agent moving in sequence to $N$ different positions according to piecewise linear walk and receiving waveforms from neighboring anchors at each position. The $N$ positions can be written as $\mathbf{P}=\left[\begin{array}{llll}\mathbf{p}_{1}^{\mathrm{T}} & \mathbf{p}_{2}^{\mathrm{T}} & \cdots & \mathbf{p}_{N}^{\mathrm{T}}\end{array}\right]^{\mathrm{T}}$, and

$$
\mathbf{R}_{k}\left(\mathbf{r}_{k j}\right)=\mathbb{E}_{\mathbf{P}}\left\{\Phi_{k j}\left(d_{k j}, d_{k j}\right) \mathbf{q}_{k j} \mathbf{q}_{k j}^{\mathrm{T}}\right\}-\mathbb{E}_{\mathbf{P}}\left\{\mathbf{q}_{k j} \boldsymbol{\Phi}_{k j}\left(d_{k j}, \mathbf{p}_{k}\right)\right\} \mathbb{E}_{\mathbf{P}}\left\{\boldsymbol{\Phi}_{k j}\left(\boldsymbol{\kappa}_{k j}, \boldsymbol{\kappa}_{k j}\right)\right\}^{-1} \mathbb{E}_{\mathbf{P}}\left\{\boldsymbol{\Phi}_{k j}\left(\mathbf{p}_{k}, d_{k j}\right) \mathbf{q}_{k j}^{\mathrm{T}}\right\}
$$

$$
\mathbf{J}_{\mathrm{e}}(\mathbf{P})=\left[\begin{array}{ccccc}
\overline{\mathbf{J}}_{\mathrm{e}}^{\mathrm{A}}\left(\mathbf{p}_{1}\right)+\sum_{j \in \mathcal{N}_{\mathrm{a}} \backslash\{1\}} \overline{\mathbf{C}}_{1, j} & -\overline{\mathbf{C}}_{1,2} & \cdots & -\overline{\mathbf{C}}_{1, N_{\mathrm{a}}} \\
-\overline{\mathbf{C}}_{1,2} & \overline{\mathbf{J}}_{\mathrm{e}}^{\mathrm{A}}\left(\mathbf{p}_{2}\right)+\sum_{j \in \mathcal{N}_{\mathrm{a}} \backslash\{2\}} \overline{\mathbf{C}}_{2, j} & & -\overline{\mathbf{C}}_{2, N_{\mathrm{a}}} \\
\vdots & & \ddots & \\
-\overline{\mathbf{C}}_{1, N_{\mathrm{a}}} & -\overline{\mathbf{C}}_{2, N_{\mathrm{a}}} & & \overline{\mathbf{J}}_{\mathrm{e}}^{\mathrm{A}}\left(\mathbf{p}_{N_{\mathrm{a}}}\right)+\sum_{j \in \mathcal{N}_{\mathrm{a}} \backslash\left\{N_{\mathrm{a}}\right\}} \overline{\mathbf{C}}_{N_{\mathrm{a}}, j}
\end{array}\right]+\mathbf{\Xi}_{\mathbf{P}}
$$


we can consider the scenario as $N$ agents in cooperation. The likelihood of the observation is

$$
f(\mathbf{r}, \hat{\mathbf{d}} \mid \boldsymbol{\theta})=\prod_{k=1}^{N} \prod_{j \in \mathcal{N}_{\mathrm{b}}} f\left(\mathbf{r}_{k j} \mid \mathbf{p}_{k}, \mathbf{p}_{j}\right) \prod_{k=1}^{N-1} f\left(\hat{d}_{k} \mid \mathbf{p}_{k}, \mathbf{p}_{k+1}\right)
$$

where $\hat{\mathbf{d}}=\left[\begin{array}{llll}\hat{d}_{1} & \hat{d}_{2} & \cdots & \hat{d}_{N-1}\end{array}\right]^{\mathrm{T}}$ in which $\hat{d}_{k}$ is the measurement of the distance $d_{k}=\left\|\mathbf{p}_{k}-\mathbf{p}_{k+1}\right\|$ between $\mathbf{p}_{k}$ and $\mathbf{p}_{k+1} \cdot{ }^{15}$ By applying Theorem 1, we have the EFIM for $\mathbf{P}$ as $\mathbf{J}_{\mathrm{e}}(\mathbf{P})=$ $\mathbf{J}_{\mathrm{e}}^{\mathrm{A}}(\mathbf{P})+\mathbf{J}_{\mathrm{e}}^{\mathrm{C}}(\mathbf{P})$ where

$$
\mathbf{J}_{\mathrm{e}}^{\mathrm{A}}(\mathbf{P})=\operatorname{diag}\left\{\mathbf{J}_{\mathrm{e}}^{\mathrm{A}}\left(\mathbf{p}_{1}\right), \mathbf{J}_{\mathrm{e}}^{\mathrm{A}}\left(\mathbf{p}_{2}\right), \ldots, \mathbf{J}_{\mathrm{e}}^{\mathrm{A}}\left(\mathbf{p}_{N}\right)\right\}
$$

and $\mathbf{J}_{\mathrm{e}}^{\mathrm{C}}(\mathbf{P})$ is given by (18), shown at the bottom of the page, in which $\mathbf{C}_{k, k+1}=\nu_{k} \mathbf{J}_{\mathrm{r}}\left(\phi_{k, k+1}\right)$ with $\phi_{k, k+1}$ denoting the angle from $\mathbf{p}_{k}$ to $\mathbf{p}_{k+1}$ and

$$
\nu_{k}=\mathbb{E}_{\hat{\mathbf{d}}}\left\{-\frac{\partial^{2}}{\partial d_{k}^{2}} \ln f\left(\hat{d}_{k} \mid \mathbf{p}_{k}, \mathbf{p}_{k+1}\right)\right\} .
$$

By further applying the notion of EFI, we can obtain the EFIM $\mathbf{J}_{\mathrm{e}}\left(\mathbf{p}_{k}\right)$ for each position $\mathbf{p}_{k}$. Note that this analysis can be extended to cooperation among multiple mobile agents over time, so that both cooperation over space and time are explored simultaneously.

3) Recursive Formula for EFIM: The structure of the EFIM in (12) and (17) enables us to extend the EFIM when agents join or leave the cooperative network. We will develop a recursive formula to construct the EFIM in the following.

Consider a network with $n$ agents in cooperation without $a$ priori knowledge of their positions, and the EFIM for agents' positions $\mathbf{J}_{\mathrm{e}}\left(\mathbf{P}_{n}\right)$ where $\mathbf{P}_{n}=\left[\begin{array}{llll}\mathbf{p}_{1}^{\mathrm{T}} & \mathbf{p}_{2}^{\mathrm{T}} & \cdots & \mathbf{p}_{n}^{\mathrm{T}}\end{array}\right]^{\mathrm{T}}$ can be obtained by (12). If a new agent enters the cooperative network, then the EFIM for the $n+1$ agents is given by

$\mathbf{J}_{\mathrm{e}}\left(\mathbf{P}_{n+1}\right)=\left[\begin{array}{cc}\mathbf{J}_{\mathrm{e}}\left(\mathbf{P}_{n}\right)+\mathbf{M}_{n, n+1} & -\mathbf{M}_{n, n+1} \mathbf{K}_{n} \\ -\mathbf{K}_{n}^{\mathrm{T}} \mathbf{M}_{n, n+1} & \mathbf{J}_{\mathrm{A}, n+1}+\mathbf{K}_{n}^{\mathrm{T}} \mathbf{M}_{n, n+1} \mathbf{K}_{n}\end{array}\right]$

where $\mathbf{J}_{\mathrm{A}, n+1}$ is the EFIM for the $(n+1)$ th agent corresponding to the localization information from anchors, $\mathbf{M}_{n, n+1}$ is the localization information from the cooperation between the $(n+$ 1)th agent and the other $n$ agents, given by

$$
\mathbf{M}_{n, n+1}=\operatorname{diag}\left\{\mathbf{C}_{1, n+1}, \mathbf{C}_{2, n+1}, \ldots, \mathbf{C}_{n, n+1}\right\}
$$

\footnotetext{
${ }^{15}$ We assume that the agent has other navigation devices, such as inertial measurement unit (IMU), odometer, or pedometer, to measure the distance between positions.
}

and $\mathbf{K}_{n} \in \mathbb{R}^{2 n \times 2}$ is given by

$$
\mathbf{K}_{n}=\left[\begin{array}{llll}
\mathbf{I}_{2 \times 2} & \mathbf{I}_{2 \times 2} & \cdots & \mathbf{I}_{2 \times 2}
\end{array}\right]^{\mathrm{T}} .
$$

Note that when the a priori knowledge of the agents' positions is available, we need to consider the contribution of $\boldsymbol{\Xi}_{\mathbf{P}}$, and the EFIM for the $n+1$ agents can be constructed in a similar way.

Similarly, when a certain agent, say $k$, leaves the network, we need to eliminate rows $2 k-1$ to $2 k$ and columns $2 k-1$ to $2 k$ in $\mathbf{J}_{\mathrm{e}}\left(\mathbf{P}_{n}\right)$, as well as subtract all corresponding $\mathbf{C}_{k j}$ for $j \in \mathcal{N}_{\mathrm{a}} \backslash\{k\}$ from the diagonal of $\mathbf{J}_{\mathrm{e}}\left(\mathbf{P}_{n}\right)$.

4) Extension to 3-D Localization: All the results obtained thus far can be easily extended to the 3-D scenario, in which $\mathbf{p}_{k}=\left[\begin{array}{lll}x_{k} & y_{k} & z_{k}\end{array}\right]^{\mathrm{T}}$. The SPEB of the $k$ th agent is defined as $\mathcal{P}\left(\mathbf{p}_{k}\right)=\left[\mathbf{J}_{\boldsymbol{\theta}}^{-1}\right]_{3 \times 3, k}$. Following the steps leading to (12) and (17), we can obtain a corresponding $3 N_{\mathrm{a}} \times 3 N_{\mathrm{a}}$ EFIM involving the RDMs $\mathbf{J}_{\mathrm{r}}\left(\varphi_{k j}, \phi_{k j}\right)$ for $k \in \mathcal{N}_{\mathrm{a}}$ and $j \in \mathcal{N}_{\mathrm{b}} \cup \mathcal{N}_{\mathrm{a}}$, where

$$
\mathbf{J}_{\mathbf{r}}(\varphi, \phi) \triangleq \mathbf{q q}^{\mathrm{T}}
$$

with $\varphi$ and $\phi$ denoting the angles in the spherical coordinates, and $\mathbf{q}=\left[\begin{array}{lll}\cos \varphi \cos \phi & \sin \varphi \cos \phi & \sin \phi\end{array}\right]^{\mathrm{T}}$.

\section{GEOMETRIC INTERPRETATION OF EFIM FOR LOCALIZATION}

In this section, we present a geometric interpretation of the EFIM for localization. This interpretation not only provides insights into the essence of localization problems, but also facilitates the analysis of localization systems, design of localization algorithms, and deployment of location-aware networks. We begin with the noncooperative case, and then extend to the cooperative case. Based on these results, we derive scaling laws of the SPEB for both noncooperative and cooperative location-aware networks.

\section{A. Interpretation for Noncooperative Localization}

When an agent only communicates with neighboring anchors, the EFIM can be written as 16

$$
\mathbf{J}_{\mathrm{e}}(\mathbf{p})=\sum_{j \in \mathcal{N}_{\mathrm{b}}} \lambda_{j} \mathbf{J}_{\mathrm{r}}\left(\phi_{j}\right) \triangleq \mathbf{U}_{\vartheta}\left[\begin{array}{cc}
\mu & 0 \\
0 & \eta
\end{array}\right] \mathbf{U}_{\vartheta}^{\mathrm{T}}
$$

where $\mu$ and $\eta$ are the eigenvalues of $\mathbf{J}_{\mathrm{e}}(\mathbf{p})$, with $\mu \geq \eta$, and $\mathbf{U}_{\vartheta}$ is a rotation matrix with angle $\vartheta$, given by

$$
\mathbf{U}_{\vartheta}=\left[\begin{array}{cc}
\cos \vartheta & -\sin \vartheta \\
\sin \vartheta & \cos \vartheta
\end{array}\right] \text {. }
$$

${ }^{16}$ To simplify the notation, we will suppress the agent's index in the subscript.

$$
\mathbf{J}_{\mathrm{e}}^{\mathrm{C}}(\mathbf{P})=\left[\begin{array}{ccccc}
\mathbf{C}_{1,2} & -\mathbf{C}_{1,2} & & & \\
-\mathbf{C}_{1,2} & \mathbf{C}_{1,2}+\mathbf{C}_{2,3} & -\mathbf{C}_{2,3} & & \\
& -\mathbf{C}_{2,3} & \ddots & \ddots & \\
& & \ddots & \mathbf{C}_{N-2, N-1}+\mathbf{C}_{N-1, N} & -\mathbf{C}_{N-1, N} \\
& & & -\mathbf{C}_{N-1, N} & \mathbf{C}_{N-1, N}
\end{array}\right]
$$




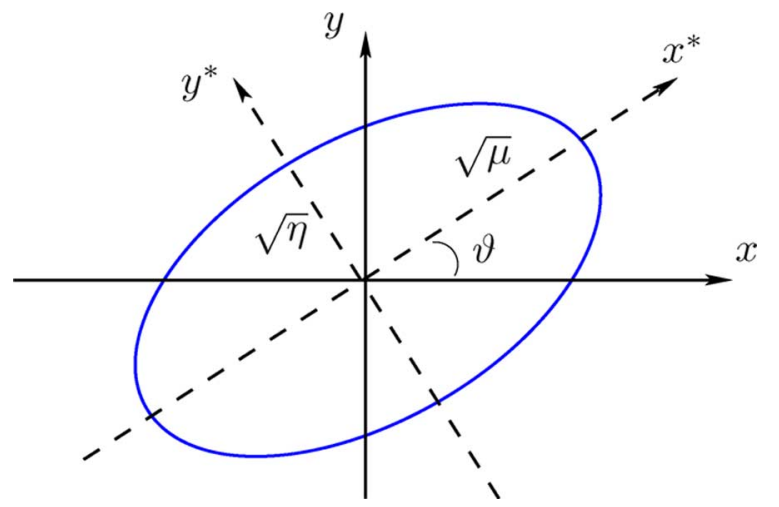

Fig. 2. Geometric interpretation of the EFIM as an information ellipse. In the rotated coordinate system (rotated over an angle $\vartheta$ ), the major and minor axes of the ellipse are given by $\sqrt{\mu}$ and $\sqrt{\eta}$, respectively.

The first and second columns of $\mathbf{U}_{\vartheta}$ are the eigenvectors corresponding to eigenvalues $\mu$ and $\eta$, respectively. By the properties of eigenvalues, we have

$$
\mu+\eta=\operatorname{tr}\left\{\mathbf{J}_{\mathrm{e}}(\mathbf{p})\right\}=\sum_{j \in \mathcal{N}_{\mathrm{b}}} \lambda_{j} .
$$

Note in (20) that $\mathbf{J}_{\mathrm{e}}(\mathbf{p})$ depends only on $\mu, \eta$, and $\vartheta$, and we will denote $\mathbf{J}_{\mathrm{e}}(\mathbf{p})$ by $\mathbf{F}(\mu, \eta, \vartheta)$ when needed.

Proposition 2: The SPEB is independent of the coordinate system.

\section{Proof: See Appendix VI.}

Remark 5: The proposition implies that if we rotate the original coordinate system by an angle $\vartheta$ prescribed by (20) and denote the agent's position in the new coordinate by $\mathbf{p}^{*}$, then the SPEB is

$$
\mathcal{P}(\mathbf{p})=\mathcal{P}\left(\mathbf{p}^{*}\right)=\operatorname{tr}\left\{\left[\begin{array}{cc}
\mu & 0 \\
0 & \eta
\end{array}\right]^{-1}\right\}=\frac{1}{\mu}+\frac{1}{\eta} .
$$

The EFIM in the new coordinate system is diagonal, and thus the localization information in these new axes is decoupled. Consequently, the SPEB is also decoupled in these two orthogonal directions.

Definition 5 (Information Ellipse): Let $\mathbf{J}$ be a $2 \times 2$ positivedefinite matrix. The information ellipse of $\mathbf{J}$ is defined as the sets of points $\mathbf{x} \in \mathbb{R}^{2}$ such that

$$
\mathbf{x} \mathbf{J}^{-1} \mathbf{x}^{\mathrm{T}}=1 .
$$

Geometrically, the EFIM in (20) corresponds to an information ellipse with major and minor axes equal to $\sqrt{\mu}$ and $\sqrt{\eta}$, respectively, and a rotation $\vartheta$ from the reference coordinate, as depicted in Fig. 2. Hence, the information ellipse is completely characterized by $\mu, \eta$, and $\vartheta$. Note that the RI is expressed as $\lambda \mathbf{J}_{\mathrm{r}}(\phi)=\mathbf{F}(\lambda, 0, \phi)$, and it corresponds to a degenerate ellipse. In the following proposition, we will show how an anchor contributes to the information ellipse of an agent.

Proposition 3: Let $\mathbf{J}_{\mathrm{e}}(\mathbf{p})=\mathbf{F}(\mu, \eta, \vartheta)$ and $\mathcal{P}(\mathbf{p})$ denote the EFIM and the SPEB of an agent, respectively. When that agent

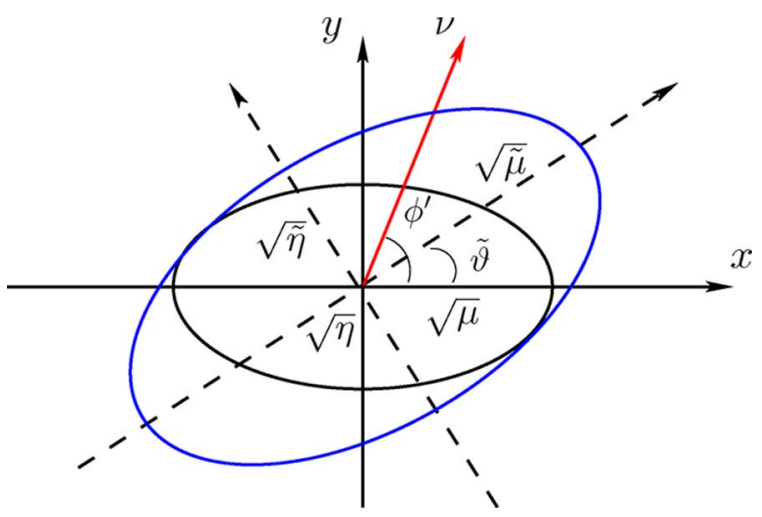

Fig. 3. Updating of the information ellipse for noncooperative localization. The original information ellipse of the agent is characterized by $\mathbf{F}(\mu, \eta, 0)$. The RI from an additional anchor is given by $\mathbf{F}(\nu, 0, \phi)$. The new information ellipse of the agent then grows along the direction $\phi^{\prime}$, but not along the orthogonal direction. The new information ellipse corresponds to $\mathbf{F}(\tilde{\mu}, \tilde{\eta}, \tilde{\vartheta})$.

obtains RI $\mathbf{F}(\nu, 0, \phi)$ from a new anchor, the new EFIM for the agent will be

$$
\begin{aligned}
\tilde{\mathbf{J}}_{\mathrm{e}}(\mathbf{p}) & =\mathbf{F}(\tilde{\mu}, \tilde{\eta}, \tilde{\vartheta}) \\
& =\mathbf{F}(\mu, \eta, \vartheta)+\mathbf{F}(\nu, 0, \phi)
\end{aligned}
$$

where the parameters for the new information ellipse are

$$
\begin{aligned}
& \tilde{\mu}=\frac{\mu+\eta+\nu}{2}+\frac{1}{2} \sqrt{\left[\mu-\eta+\nu \cos 2 \phi^{\prime}\right]^{2}+\nu^{2} \sin ^{2} 2 \phi^{\prime}} \\
& \tilde{\eta}=\frac{\mu+\eta+\nu}{2}-\frac{1}{2} \sqrt{\left[\mu-\eta+\nu \cos 2 \phi^{\prime}\right]^{2}+\nu^{2} \sin ^{2} 2 \phi^{\prime}}
\end{aligned}
$$

and

$$
\tilde{\vartheta}=\vartheta+\frac{1}{2} \arctan \frac{\nu \sin 2 \phi^{\prime}}{\mu-\eta+\nu \cos 2 \phi^{\prime}}
$$

with $\phi^{\prime} \triangleq \phi-\vartheta$. Correspondingly, the new SPEB becomes

$$
\tilde{\mathcal{P}}(\mathbf{p})=\frac{1}{\tilde{\mu}}+\frac{1}{\tilde{\eta}}=\frac{\mu+\eta+\nu}{\mu \eta+\nu\left[\eta+(\mu-\eta) \sin ^{2} \phi^{\prime}\right]} .
$$

Remark 6: The geometric interpretation for the proposition is depicted in Fig. 3. For a fixed RII $\nu$, we see from (21) that $\tilde{\mathcal{P}}(\mathbf{p})$ can be minimized through $\phi^{\prime}$ (equivalently, through $\phi$ ) in the denominator, leading to

$$
\min _{\phi} \tilde{\mathcal{P}}(\mathbf{p})=\frac{\mu+\eta+\nu}{\mu(\eta+\nu)}
$$

and the minimum is achieved when $\phi=\vartheta \pm \pi / 2$. In such a case, the anchor is along the direction of the eigenvector corresponding to the smallest eigenvalue $\eta$. Observe also that the denominator in (21) is equal to $\tilde{\mu} \cdot \tilde{\eta}$, which is proportional to the squared area of the new information ellipse corresponding to $\tilde{\mathbf{J}}_{\mathrm{e}}(\mathbf{p})$. Hence, for a fixed $\nu$, the minimum SPEB is achieved when the new anchor is along the minor axis of the information ellipse corresponding to $\mathbf{J}_{\mathrm{e}}(\mathbf{p})$. Equivalently, this choice of anchor position maximizes the area of the new information ellipse.

On the other hand, the maximum SPEB occurs when the anchor is along the direction of the eigenvector corresponding to 
the largest eigenvalue $\mu$, i.e., the major axis of the information ellipse corresponding to $\mathbf{J}_{\mathrm{e}}(\mathbf{p})$. Equivalently, this minimizes the area of the new information ellipse, and thus

$$
\max _{\phi} \tilde{\mathcal{P}}(\mathbf{p})=\frac{\mu+\eta+\nu}{\eta(\mu+\nu)}
$$

and the maximum is achieved when $\phi=\vartheta \pm \pi$. Note also that

$$
\frac{1}{\mu}<\tilde{\mathcal{P}}(\mathbf{p}) \leq \mathcal{P}(\mathbf{p})
$$

where the left-hand side $1 / \mu=\lim _{\nu \rightarrow \infty} \min _{\phi} \tilde{\mathcal{P}}(\mathbf{p})$, and the right-hand side $\mathcal{P}(\mathbf{p})=\lim _{\nu \rightarrow 0} \tilde{\mathcal{P}}(\mathbf{p})$.

\section{B. Interpretation for Cooperative Localization}

The EFIM for all the agents in cooperative location-aware network is given, respectively, by (17) and (12) for the cases with and without a priori position knowledge. Further applying the notion of EFI, one can obtain the EFIM for individual agents. In general, the exact EFIM expression for the individual agents is complicated. However, we can find lower and upper bounds on the individual EFIM to gain some insights into the localization problem.

Proposition 4: Let $\mathbf{J}_{\mathrm{e}}^{\mathrm{A}}\left(\mathbf{p}_{k}\right)=\mathbf{F}\left(\mu_{k}, \eta_{k}, \vartheta_{k}\right)$ denote the EFIM for agent $k$ that corresponds to the localization information from anchors, and let $\mathbf{C}_{k j}=\mathbf{F}\left(\nu_{k j}, 0, \phi_{k j}\right)$ denote the RI for that agent obtained from cooperation with agent $j$. The EFIM $\mathbf{J}_{\mathrm{e}}\left(\mathbf{p}_{k}\right)$ for agent $k$ can be bounded as follows:

$$
\mathbf{J}_{\mathrm{e}}^{\mathrm{L}}\left(\mathbf{p}_{k}\right) \preceq \mathbf{J}_{\mathrm{e}}\left(\mathbf{p}_{k}\right) \preceq \mathbf{J}_{\mathrm{e}}^{\mathrm{U}}\left(\mathbf{p}_{k}\right)
$$

where

$$
\begin{aligned}
& \mathbf{J}_{\mathrm{e}}^{\mathrm{L}}\left(\mathbf{p}_{k}\right)=\mathbf{J}_{\mathrm{e}}^{\mathrm{A}}\left(\mathbf{p}_{k}\right)+\sum_{j \in \mathcal{N}_{\mathrm{a}} \backslash\{k\}} \xi_{k j}^{\mathrm{L}} \mathbf{C}_{k j} \\
& \mathbf{J}_{\mathrm{e}}^{\mathrm{U}}\left(\mathbf{p}_{k}\right)=\mathbf{J}_{\mathrm{e}}^{\mathrm{A}}\left(\mathbf{p}_{k}\right)+\sum_{j \in \mathcal{N}_{\mathrm{a}} \backslash\{k\}} \xi_{k j}^{\mathrm{U}} \mathbf{C}_{k j}
\end{aligned}
$$

with coefficients $0 \leq \xi_{k j}^{\mathrm{L}} \leq \xi_{k j}^{\mathrm{U}} \leq 1$ given by (44) and (46).

Proof: See Appendix VI.

Remark 7: The bounds for the EFIM can be written as weighted sums of RIs from the neighboring nodes, and such linear forms can facilitate analysis and design of location-aware networks. Moreover, it turns out that $\xi_{k j}^{\mathrm{L}}=\xi_{k j}^{\mathrm{U}}$ when there are only two agents in cooperation, leading to the following corollary.

Corollary 2: Let $\mathbf{J}_{\mathrm{e}}^{\mathrm{A}}\left(\mathbf{p}_{1}\right)=\mathbf{F}\left(\mu_{1}, \eta_{1}, \vartheta_{1}\right)$ and $\mathbf{J}_{\mathrm{e}}^{\mathrm{A}}\left(\mathbf{p}_{2}\right)=$ $\mathbf{F}\left(\mu_{2}, \eta_{2}, \vartheta_{2}\right)$ denote the EFIMs for agents 1 and 2 from anchors, respectively, and let $\mathbf{C}_{1,2}=\mathbf{F}\left(\nu_{1,2}, 0, \phi_{1,2}\right)$ denote the RI from their cooperation. The EFIMs for the two agents are given, respectively, by (see also Fig. 4)

and

$$
\mathbf{J}_{\mathrm{e}}\left(\mathbf{p}_{1}\right)=\mathbf{J}_{\mathrm{e}}^{\mathrm{A}}\left(\mathbf{p}_{1}\right)+\xi_{1,2} \nu_{1,2} \mathbf{J}_{\mathrm{r}}\left(\phi_{1,2}\right)
$$

$$
\mathbf{J}_{\mathrm{e}}\left(\mathbf{p}_{2}\right)=\mathbf{J}_{\mathrm{e}}^{\mathrm{A}}\left(\mathbf{p}_{2}\right)+\xi_{2,1} \nu_{1,2} \mathbf{J}_{\mathrm{r}}\left(\phi_{1,2}\right)
$$

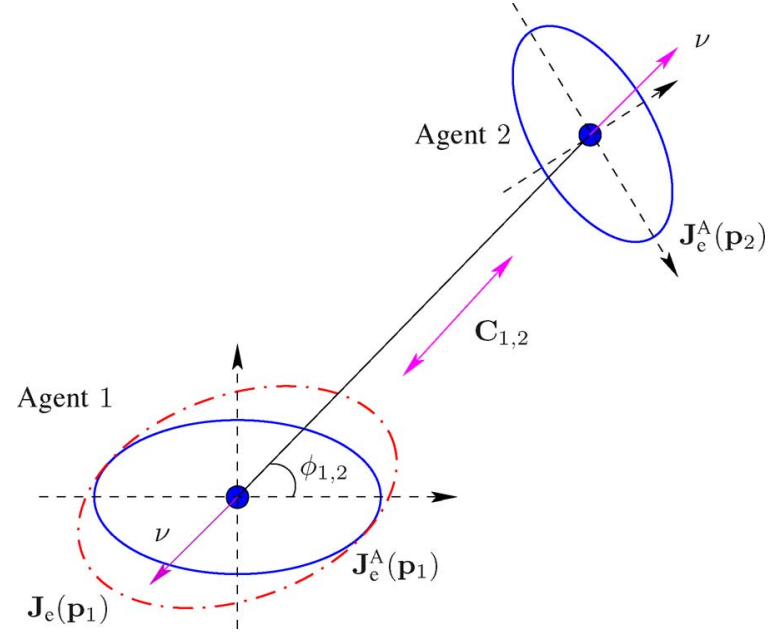

Fig. 4. Updating of the information ellipse for cooperative localization. Based on the anchors, the $k$ th agent has information $\mathbf{J}_{\mathrm{e}}^{\mathrm{A}}\left(\mathbf{p}_{k}\right)$. The cooperative information between the two agents is given by $\mathbf{C}_{1,2}=\mathbf{F}\left(\nu, 0, \phi_{1,2}\right)$. The total EFIM for agent 1 is then $\mathbf{J}_{\mathrm{e}}\left(\mathbf{p}_{1}\right)=\mathbf{J}_{\mathrm{e}}^{\mathrm{A}}\left(\mathbf{p}_{1}\right)+\xi_{1,2} \mathbf{C}_{1,2}$. The new information ellipse grows along the line connecting the two agents.

where

and

$$
\xi_{1,2}=\frac{1}{1+\nu_{1,2} \Delta_{2}\left(\phi_{1,2}\right)}
$$

$$
\xi_{2,1}=\frac{1}{1+\nu_{1,2} \Delta_{1}\left(\phi_{1,2}\right)}
$$

with

$$
\Delta_{k}\left(\phi_{1,2}\right)=\mathbf{q}_{12}^{\mathrm{T}}\left[\mathbf{J}_{\mathrm{e}}^{\mathrm{A}}\left(\mathbf{p}_{k}\right)\right]^{-1} \mathbf{q}_{12}
$$

for $k=1,2$.

Remark 8: The results follow directly from Proposition 4. We make the following remarks.

- Cooperation provides agent 1 with RI $\xi_{1,2} \nu_{1,2} \mathbf{J}_{\mathrm{r}}\left(\phi_{1,2}\right)$ with $0 \leq \xi_{1,2} \leq 1$. Hence, agent 1 obtains an RII $\xi_{1,2} \nu_{1,2}$ from cooperation instead of the full RII $\nu_{1,2}$. This degradation in RII is due to the inherent uncertainty of the second agent's position. We introduce the effective RII $\tilde{\nu}_{1,2}=$ $\xi_{1,2} \nu_{1,2}$.

- The effective RII has the following geometric interpretation. The value $\Delta_{2}\left(\phi_{1,2}\right)$ is the DPEB of agent 2 (based solely on the anchors) along the angle $\phi_{1,2}$ between the two agents. This implies that the larger the uncertainty of agent 2 along the angle $\phi_{1,2}$, the less effective cooperation is. For a given $\Delta_{2}\left(\phi_{1,2}\right)$, the effective RII $\tilde{\nu}_{1,2}$ increases monotonically with $\nu_{1,2}$, and has the following asymptotic limits:

$$
\begin{aligned}
\lim _{\nu_{1,2} \rightarrow 0} \tilde{\nu}_{1,2} & =0 \\
\lim _{\nu_{1,2} \rightarrow \infty} \tilde{\nu}_{1,2} & =1 / \Delta_{2}\left(\phi_{1,2}\right) .
\end{aligned}
$$


Hence, the maximum effective RII that agent 2 can provide to agent 1 equals the inverse of the DPEB of agent 2 (based solely on the anchors) along the angle $\phi_{1,2}$ between the two agents.

- When i) the two agents happen to be oriented such that $\phi_{1,2}=\vartheta_{2}$, and ii) agent 2 is certain about its position along that angle $\left(\mu_{2}=+\infty\right)$, then $\Delta_{2}\left(\phi_{1,2}\right)=0$ and $\mathbf{J}_{\mathrm{e}}\left(\mathbf{p}_{1}\right)=\mathbf{J}_{\mathrm{e}}^{\mathrm{A}}\left(\mathbf{p}_{1}\right)+\mathbf{C}_{1,2}$, i.e., agent 2 can be thought of as an anchor from the standpoint of providing RI to agent 1. From this perspective, anchors and agents are equivalent for localization, where anchors are special agents with zero $\mathrm{SPEB}$, or equivalently, infinite $\mathbf{J}_{\mathrm{e}}^{\mathrm{A}}\left(\mathbf{p}_{k}\right)$ in all directions.

\section{Scaling Laws for Location-Aware Networks}

In this section, we derive scaling laws of the SPEB for both noncooperative and cooperative location-aware networks. Scaling laws give us insight into the benefit of cooperation for localization in large networks. As we will see, agents and anchors contribute equally to the scaling laws for cooperative location-aware networks.

We focus on two types of random networks: dense networks and extended networks [47], [48]. In both types of networks, we consider the $N_{\mathrm{b}}$ anchors and $N_{\mathrm{a}}$ agents randomly located (uniformly distributed) in the plane. In dense networks, adding nodes increases the node density, while the area remains constant. In extended networks, the area increases proportional to the number of nodes, while both the anchor and the agent densities remain constant. Without loss of generality, we consider one round of transmission from each node to another. All transmission powers are the same, while large- and small-scale fading can be arbitrary. Medium access control is assumed so that these signals do not interfere with one another.

Definition 6 (Scaling of SPEB): Consider a network with $n$ nodes randomly located in a given area. We say that the SPEB of individual agents scales as $\Theta(f(n))$ for some function $f(n)$, denoted by $\mathcal{P}(\mathbf{p}) \in \Theta(f(n))$, if there are deterministic constants $0<c_{1}<c_{2}<+\infty$ such that

$$
\mathbb{P}\left\{c_{1} f(n) \leq \mathcal{P}(\mathbf{p}) \leq c_{2} f(n)\right\}=1-\epsilon(n)
$$

where $\lim _{n \rightarrow \infty} \epsilon(n)=0$.

Theorem 5: In dense networks, the SPEB of each agent scales as $\Theta\left(1 / N_{\mathrm{b}}\right)$ for noncooperative localization, and as $\Theta\left(1 /\left(N_{\mathrm{b}}+\right.\right.$ $\left.N_{\mathrm{a}}\right)$ ) for cooperative localization.

Proof: See Appendix VII.

Theorem 6: In extended networks with an amplitude loss exponent $b,{ }^{17}$ the SPEB of each agent scales as

$$
\mathcal{P}(\mathbf{p}) \in \begin{cases}\Theta\left(1 / \log N_{\mathrm{b}}\right), & b=1 \\ \Theta(1), & b>1 \\ \Theta\left(1 / N_{\mathrm{b}}^{b-1}\right), & 0<b<1\end{cases}
$$

\footnotetext{
${ }^{17}$ Note that the amplitude loss exponent is $b$, while the corresponding power loss exponent is $2 b$. The amplitude loss exponent $b$ is environment dependent and can range from approximately 0.8 (e.g., hallways inside buildings) to 4 (e.g., dense urban environments) [49].
}

for noncooperative localization, and

$$
\mathcal{P}(\mathbf{p}) \in \begin{cases}\Theta\left(1 / \log \left(N_{\mathrm{b}}+N_{\mathrm{a}}\right)\right), & b=1 \\ \Theta(1), & b>1 \\ \Theta\left(1 /\left(N_{\mathrm{b}}+N_{\mathrm{a}}\right)^{b-1}\right), & 0<b<1\end{cases}
$$

for cooperative localization.

Proof: See Appendix VII.

Remark 9: We make the following remarks.

- In dense networks, the SPEB scales inversely proportional to the number of anchors for noncooperative localization, and inversely proportional to the number of nodes for cooperative localization. The gain from cooperation is given by $\Theta\left(1+N_{\mathrm{a}} / N_{\mathrm{b}}\right)$, and hence the benefit is most pronounced when the number of anchors is limited. Moreover, it is proven in Appendix VII that $\epsilon(n)$ decreases exponentially with the number of nodes.

- In extended networks with an amplitude loss exponent equal to 1, the SPEB scales inversely proportional to the logarithm of the number of anchors for noncooperative localization, and inversely proportional to the logarithm of the number of nodes for cooperative localization. This implies that the SPEB in extended networks decreases much more slowly than that in dense networks, and the gain from cooperation is now reduced to $\Theta\left(\log \left(N_{\mathrm{b}}+N_{\mathrm{a}}\right) / \log N_{\mathrm{b}}\right)$. Moreover, it is shown in Appendix VII that $\epsilon(n)$ decreases as $\exp \left(-(\log n)^{2} / 8\right) / \log n$.

- In extended networks with an amplitude loss exponent greater than 1, the SPEB converges to a strict positive value as the network grows. This agrees with our intuition that as more nodes are added, the benefit of the additional nodes diminishes due to the rapidly decaying RII provided by those nodes. It can be shown that the SPEB converges to a smaller value in the cooperative case than that in the noncooperative case, i.e., a constant gain can be obtained by cooperation.

\section{NUMERICAL RESULTS}

In this section, we examine several numerical examples pertaining to cooperative localization and illustrate practical applications of our analytical results.

\section{A. Effective RI}

We first investigate the behavior of the effective RII $\tilde{\nu}_{1,2}$ from Corollary 2 when two agents cooperate. The effective RII $\tilde{\nu}_{1,2}$ is plotted in Fig. 5 as a function of the RII $\nu_{1,2}$ for $\mathbf{J}_{\mathrm{e}}^{\mathrm{A}}\left(\mathbf{p}_{2}\right)=$ $\mathbf{F}\left(\mu_{2}=2, \eta_{2}=1, \vartheta_{2}=0\right)$ and various values of $\phi_{1,2}$. The corresponding asymptotic limits are also plotted for large values of $\nu_{1,2}$. We observe that effective RII increases from 0 to $1 / \Delta_{2}\left(\phi_{1,2}\right)$ as the RII $\nu_{1,2}$ increases. For a fixed RII, the second agent will provide the maximum effective RII at $\phi_{1,2}=\vartheta_{2}$, along which angle the second agent has the minimum DPEB (i.e., $\left.1 / \mu_{2}=0.5\right)$. On the other hand, the second agent will provide the minimum effective RII at $\phi_{1,2}=\vartheta_{2} \pm \pi / 2$, along which angle the second agent has the maximum DPEB (i.e., $1 / \eta_{2}=1$ ).

\section{B. Benefit of Cooperation}

We now consider the SPEB performance as a function of the number of agents for cooperative localization. The network 


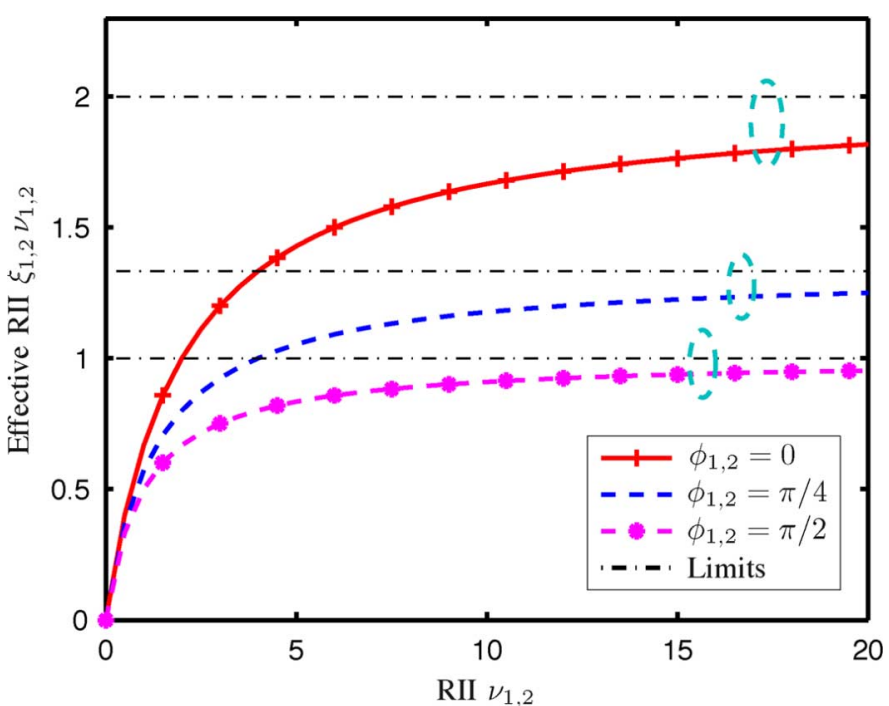

Fig. 5. Effective RII $\xi_{1,2} \nu_{1,2}$ as a function of the RII $\nu_{1,2}$, for $\mathbf{J}_{\mathrm{A}, 2}=$ $\mathbf{F}\left(\mu_{2}=2, \eta_{2}=1, \vartheta_{2}=0\right)$, and different angle of arrival $\phi_{1,2}$.

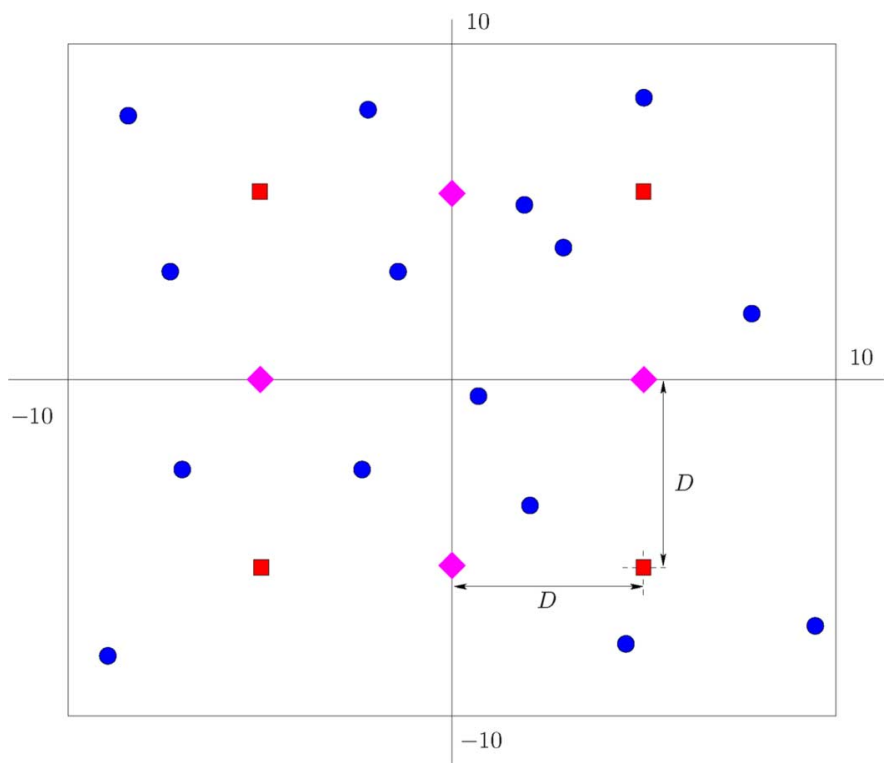

Fig. 6. Typical network deployment of two sets of anchors (set I: squares, set II: diamonds) and $N_{\mathrm{a}}=15$ agents. The agents are distributed uniformly over the $[-10,10] \times[-10,10]$ map, while the locations of the anchors are controlled by $D$.

configuration is shown in Fig. 6. The agents randomly (uniformly distributed) reside in a $20 \mathrm{~m}$ by $20 \mathrm{~m}$ area. There are two sets of anchors [shown as squares (set I) and diamonds (set II) in Fig. 6], with a configuration determined by the parameter $D$. Since fading does not affect the scaling behavior as shown Section IV-C, we consider a network with signals that obey the free-space path-loss model for simplicity, so that the RII $\lambda_{k j} \propto 1 / d_{k j}^{2}$.

Fig. 7 shows the average SPEB over all the agents as a function of the number of agents, obtained by Monte Carlo simulation, for $D=10$. We see that as the number of agents increases, the average SPEB decreases significantly, roughly proportional to the number of agents. Note that the anchor configuration set II yields a lower SPEB. Intuitively, this is due to the fact that

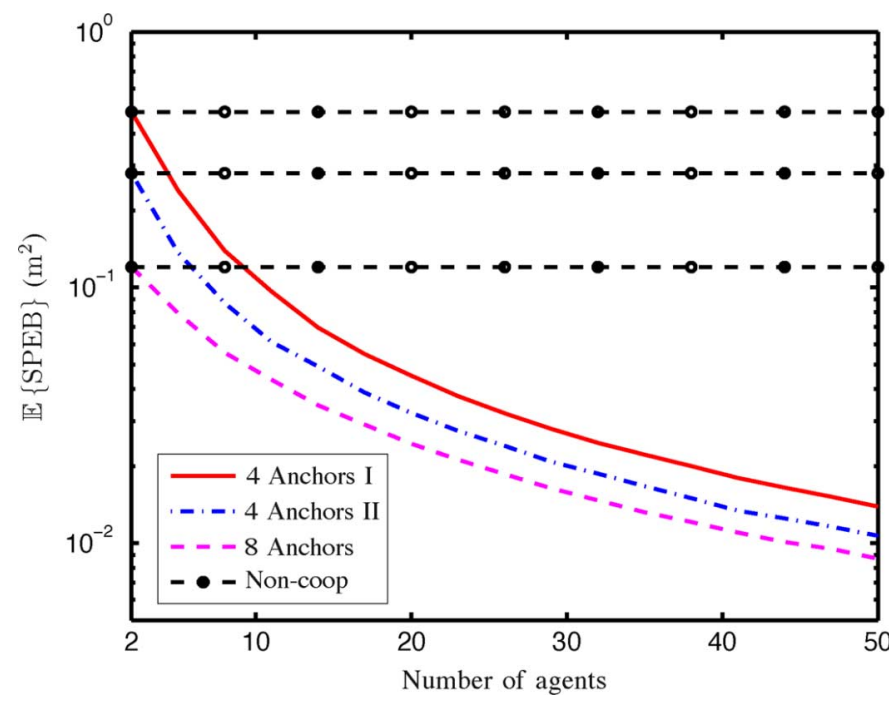

Fig. 7. The average SPEB as a function of the number of agents in the network for various anchor configurations $(D=10)$.

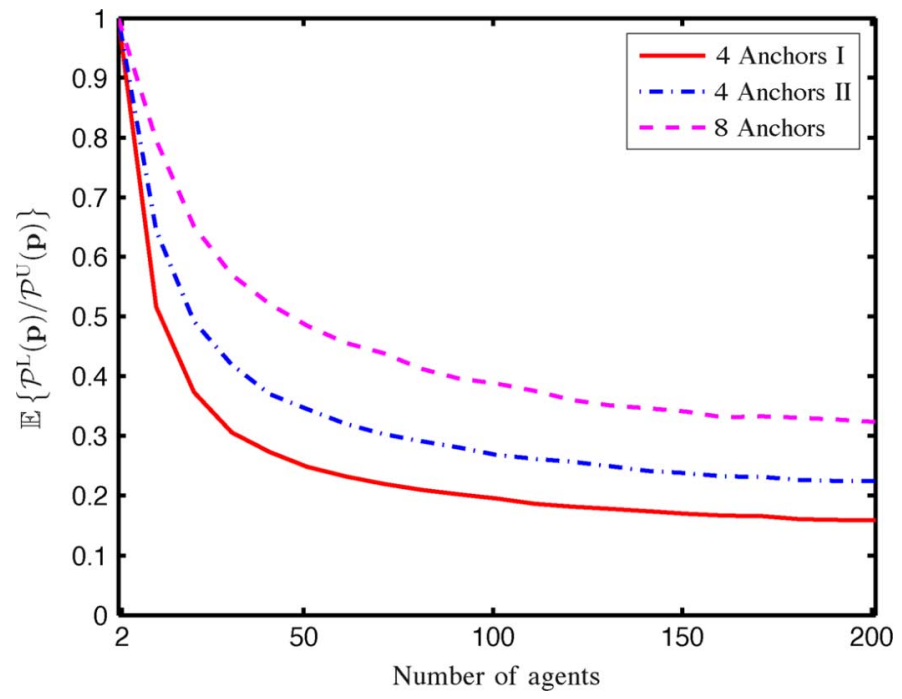

Fig. 8. Ratio of upper and lower approximations of the $\operatorname{SPEB}, \mathcal{P} \mathrm{L}(\mathbf{p})$ and $\mathcal{P} \mathrm{U}(\mathbf{p})$, as a function of the number of agents for anchor set I, set II, and both.

the anchors in set II (distance $D$ from the center) cover the area better than the anchors in set I (distance $\sqrt{2} D$ from the center).

Define the upper and lower approximations of agent $k$ 's SPEB as

$$
\mathcal{P}^{\mathrm{U}}\left(\mathbf{p}_{k}\right) \triangleq \operatorname{tr}\left\{\left[\mathbf{J}_{\mathrm{e}}^{\mathrm{L}}\left(\mathbf{p}_{k}\right)\right]^{-1}\right\}
$$

and

$$
\mathcal{P}^{\mathrm{L}}\left(\mathbf{p}_{k}\right) \triangleq \operatorname{tr}\left\{\left[\mathbf{J}_{\mathrm{e}}^{\mathrm{U}}\left(\mathbf{p}_{k}\right)\right]^{-1}\right\}
$$

where $\mathbf{J}_{\mathrm{e}}^{\mathrm{L}}\left(\mathbf{p}_{k}\right)$ and $\mathbf{J}_{\mathrm{e}}^{\mathrm{U}}\left(\mathbf{p}_{k}\right)$ are given by (22) and (23), respectively, in Theorem 4. Fig. 8 shows the average ratio of the lower and upper approximations of the SPEB, obtained by Monte Carlo simulation, for anchor set I, set II, and both sets. When there are only two agents in cooperation, the bounds coincide, as we expect from Corollary 2. As the number of 


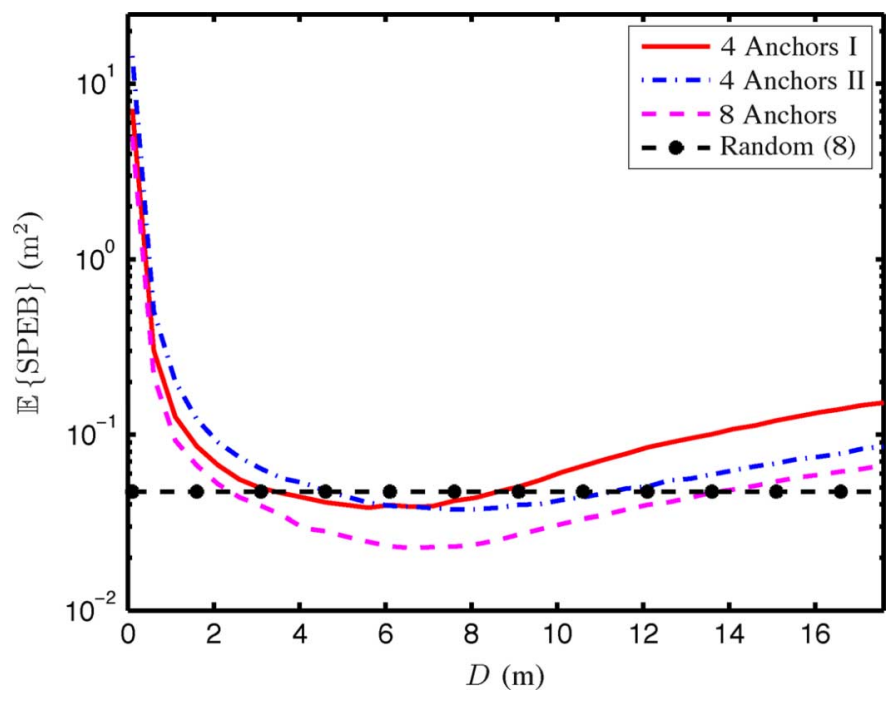

Fig. 9. The mean SPEB with respective to anchor deployment. There are $N_{\mathrm{a}}=$ 15 agents.

agents increases, the ratio deviates from 1 , or equivalently, the approximations become looser, due to the fact that upper approximation ignores more cooperative information, and the lower approximation considers more agents to be equivalent to anchors. Nevertheless, the ratio converges to a positive constant, implying that the upper and lower approximations decrease at the same rate in an asymptotical regime, as shown in the proof of Theorem 5 .

\section{Anchor Deployment}

Finally, we investigate the effect of anchor deployment in more detail. We consider a scenario with $N_{\mathrm{a}}=15$ agents. The anchor placement is controlled through $D$ (see Fig. 6). Fig. 9 shows the average SPEB as a function of $D$ for different anchor configurations (set I, set II, and both sets). We see that the SPEB first decreases, and then increases, as a function of $D$. When $D$ is close to 0 , all the anchors are located closely in the middle of the area, and hence the RIs from those anchors to a particular agent are nearly in the same direction. This will greatly increase the error of each agent's position since every $\mathbf{J}_{\mathrm{e}}^{\mathrm{A}}\left(\mathbf{p}_{k}\right)$ is close to singular, resulting in poor overall SPEB performance. As the anchors begin to move away from the center, they provide RIs along different directions to each agent, which lowers the average SPEB. Then, as the distances of the anchors to the center increase further, the anchors become far away from more and more agents. Hence, the RII decreases due to the path-loss phenomena, and this leads to the increase in the average SPEB. Observe also that anchor set I is better than anchor set II for $D<$ $7 \mathrm{~m}$. This is because, for a fixed $D<7 \mathrm{~m}$, anchor set I can cover a larger area. For $D>7 \mathrm{~m}$, anchor set I suffers more from path loss than anchor set II.

For the sake of comparison, we have also included the average SPEB when eight anchors are deployed 1) according to set I and II simultaneously, and 2) randomly in a $[-10 \mathrm{~m}, 10 \mathrm{~m}] \times$ $[-10 \mathrm{~m}, 10 \mathrm{~m}]$ area. The figure shows that intelligent anchor deployment can be beneficial compared to random deployment, indicating the need for anchor deployment strategies.

\section{CONCLUSION}

In this paper, we have investigated the fundamental limits on the localization accuracy for wideband cooperative location-aware networks. We have derived the SPEB by applying the notion of EFI to characterize the localization accuracy. Since our analysis exploits the received waveforms rather than specific signal metrics, the SPEB incorporates all the localization information inherent in the received waveforms. Our methodology unifies the localization information from anchors and that from cooperation among agents in a canonical form, viz. RI, and the total localization information is a sum of these individual RIs. We have put forth a geometrical interpretation of the EFIM based on eigendecomposition, and this interpretation has facilitated the theoretical analysis of the localization information for cooperative networks. We have also derived scaling laws for the SPEB in both dense and extended networks, showing the benefit of cooperation in an asymptotic regime. Our results provide fundamental new insights into the essence of the localization problem, and can be used as guidelines for localization system design as well as benchmarks for cooperative location-aware networks.

\section{APPENDIX I}

PROOF OF PROPOSITION 1

Proof: The right-hand side of (5) can be written as

$$
\begin{aligned}
\mathcal{P} & \left(\mathbf{p}_{k} ; \mathbf{u}\right)+\mathcal{P}\left(\mathbf{p}_{k} ; \mathbf{u}_{\perp}\right) \\
& =\operatorname{tr}\left\{\mathbf{u}^{\mathrm{T}}\left[\mathbf{J}_{\boldsymbol{\theta}}^{-1}\right]_{2 \times 2, k} \mathbf{u}\right\}+\operatorname{tr}\left\{\mathbf{u}_{\perp}^{\mathrm{T}}\left[\mathbf{J}_{\boldsymbol{\theta}}^{-1}\right]_{2 \times 2, k} \mathbf{u}_{\perp}\right\} \\
& =\operatorname{tr}\left\{\left[\mathbf{J}_{\boldsymbol{\theta}}^{-1}\right]_{2 \times 2, k} \mathbf{u} \mathbf{u}^{\mathrm{T}}\right\}+\operatorname{tr}\left\{\left[\mathbf{J}_{\boldsymbol{\theta}}^{-1}\right]_{2 \times 2, k} \mathbf{u}_{\perp} \mathbf{u}_{\perp}^{\mathrm{T}}\right\} \\
& =\operatorname{tr}\left\{\left[\mathbf{J}_{\boldsymbol{\theta}}^{-1}\right]_{2 \times 2, k}\right\}=\mathcal{P}\left(\mathbf{p}_{k}\right)
\end{aligned}
$$

where we have used the fact $\mathbf{u}^{\mathrm{T}}+\mathbf{u}_{\perp} \mathbf{u}_{\perp}^{\mathrm{T}}=\mathbf{I}$.

\section{APPENDIX II \\ PROOF OF THEOREM 1}

We proceed in two steps: we first show that the EFIM is structured as in (12), and then derive the details of the RI.

\section{A. Derivation of the EFIM Structure}

When a priori knowledge of the agents' positions is unavailable, the log-likelihood function in (10) becomes

$$
\begin{array}{r}
\ln f(\mathbf{r}, \boldsymbol{\kappa} \mid \mathbf{P})=\sum_{k \in \mathcal{N}_{\mathrm{a}}} \sum_{j \in \mathcal{N}_{\mathrm{b}} \cup \mathcal{N}_{\mathrm{a}} \backslash\{k\}}\left[\ln f\left(\mathbf{r}_{k j} \mid \mathbf{p}_{k}, \mathbf{p}_{j}, \boldsymbol{\kappa}_{k j}\right)\right. \\
\left.+\ln f\left(\boldsymbol{\kappa}_{k j} \mid \mathbf{p}_{k}, \mathbf{p}_{j}\right)\right]
\end{array}
$$

where $\boldsymbol{\kappa}$ denotes the vector of the channel parameters containing all $\boldsymbol{\kappa}_{k j}$ with $k \in \mathcal{N}_{\mathrm{a}}$ and $j \in \mathcal{N}_{\mathrm{b}} \cup \mathcal{N}_{\mathrm{a}} \backslash\{k\}$. For notational convenience, we now introduce

$$
\begin{aligned}
& \mathbf{\Phi}(\mathbf{x}, \mathbf{y}) \triangleq \mathbb{E}_{\mathbf{r}, \boldsymbol{\kappa}}\left\{-\frac{\partial^{2} \ln f(\mathbf{r}, \boldsymbol{\kappa} \mid \mathbf{P})}{\partial \mathbf{x} \partial \mathbf{y}^{\mathrm{T}}}\right\} \\
& \boldsymbol{\Phi}_{k j}(\mathbf{x}, \mathbf{y}) \triangleq \mathbb{E}_{\mathbf{r}, \boldsymbol{\kappa}}\left\{-\frac{\partial^{2}}{\partial \mathbf{x} \partial \mathbf{y}^{\mathrm{T}}}\left[\ln f\left(\mathbf{r}_{k j} \mid \mathbf{p}_{k}, \mathbf{p}_{j}, \boldsymbol{\kappa}_{k j}\right)\right.\right. \\
&\left.\left.+\ln f\left(\boldsymbol{\kappa}_{k j} \mid \mathbf{p}_{k}, \mathbf{p}_{j}\right)\right]\right\}
\end{aligned}
$$


as well as

$$
\begin{aligned}
& \Upsilon(\mathrm{x}, \mathrm{y}, \mathrm{z}) \triangleq \boldsymbol{\Phi}(\mathrm{x}, \mathrm{y})[\boldsymbol{\Phi}(\mathrm{y}, \mathrm{y})]^{-1} \boldsymbol{\Phi}(\mathrm{y}, \mathrm{z}) \\
& \boldsymbol{\Upsilon}_{k j}(\mathbf{x}, \mathbf{y}, \mathbf{z}) \triangleq \boldsymbol{\Phi}_{k j}(\mathbf{x}, \mathbf{y})\left[\boldsymbol{\Phi}_{k j}(\mathbf{y}, \mathbf{y})\right]^{-1} \boldsymbol{\Phi}_{k j}(\mathbf{y}, \mathbf{z}) \text {. }
\end{aligned}
$$

Since $\boldsymbol{\Phi}\left(\tilde{\boldsymbol{\theta}}_{k}, \tilde{\boldsymbol{\theta}}_{j}\right)=\mathbf{0}$ for $k \neq j$, the EFIM for $\mathbf{P}$ can be derived as

$$
\mathbf{J}_{\mathrm{e}}(\mathbf{P})=\boldsymbol{\Phi}(\mathbf{P}, \mathbf{P})-\sum_{k \in \mathcal{N}_{\mathrm{a}}} \boldsymbol{\Upsilon}\left(\mathbf{P}, \tilde{\boldsymbol{\theta}}_{k}, \mathbf{P}\right)
$$

Structure of $\boldsymbol{\Phi}(\mathbf{P}, \mathbf{P})$ : Due to the structure in (25), we can express $\boldsymbol{\Phi}(\mathbf{P}, \mathbf{P})$ as

$$
\begin{aligned}
\boldsymbol{\Phi}(\mathbf{P}, \mathbf{P}) & =\sum_{k \in \mathcal{N}_{\mathrm{a}}} \sum_{j \in \mathcal{N}_{\mathrm{b}}} \boldsymbol{\Phi}_{k j}(\mathbf{P}, \mathbf{P})+\sum_{k \in \mathcal{N}_{\mathrm{a}}} \sum_{j \in \mathcal{N}_{\mathrm{a}} \backslash\{k\}} \boldsymbol{\Phi}_{k j}(\mathbf{P}, \mathbf{P}) \\
& \triangleq \mathbf{K}_{\mathrm{A}}+\mathbf{K}_{\mathrm{C}}
\end{aligned}
$$

where $\mathbf{K}_{\mathrm{A}} \in \mathbb{R}^{2 N_{\mathrm{a}} \times 2 N_{\mathrm{a}}}$ is a block-diagonal matrix, consisting of $2 \times 2$ block matrices, given by

$$
\left[\mathbf{K}_{\mathrm{A}}\right]_{2 k-1: 2 k, 2 m-1: 2 m}= \begin{cases}\sum_{j \in \mathcal{N}_{\mathrm{b}}} \mathbf{\Phi}_{k j}\left(\mathbf{p}_{k}, \mathbf{p}_{k}\right), & k=m \\ \mathbf{0}, & k \neq m .\end{cases}
$$

On the other hand, $\mathbf{K}_{\mathrm{C}} \in \mathbb{R}^{2 N_{\mathrm{a}} \times 2 N_{\mathrm{a}}}$ is also a block matrix, consisting of $2 \times 2$ block matrices, given by (29), shown at the bottom of the page.

Structure of $\boldsymbol{\Upsilon}\left(\mathbf{P}, \tilde{\boldsymbol{\theta}}_{k}, \mathbf{P}\right)$ : Since $\boldsymbol{\Phi}\left(\boldsymbol{\kappa}_{k i}, \boldsymbol{\kappa}_{k j}\right)=\mathbf{0}$ for $i \neq j$, we find that

$$
\begin{aligned}
\sum_{k \in \mathcal{N}_{\mathrm{a}}} \boldsymbol{\Upsilon}\left(\mathbf{P}, \tilde{\boldsymbol{\theta}}_{k}, \mathbf{P}\right)= & \sum_{k \in \mathcal{N}_{\mathrm{a}}} \sum_{j \in \mathcal{N}_{\mathrm{b}}} \boldsymbol{\Upsilon}_{k j}\left(\mathbf{P}, \boldsymbol{\kappa}_{k j}, \mathbf{P}\right) \\
& +\sum_{k \in \mathcal{N}_{\mathrm{a}}} \sum_{j \in \mathcal{N}_{\mathrm{a}} \backslash\{k\}} \boldsymbol{\Upsilon}_{k j}\left(\mathbf{P}, \boldsymbol{\kappa}_{k j}, \mathbf{P}\right) \\
\triangleq & \mathbf{M}_{\mathrm{A}}+\mathbf{M}_{\mathrm{C}}
\end{aligned}
$$

where $\mathbf{M}_{\mathrm{A}} \in \mathbb{R}^{2 N_{\mathrm{a}} \times 2 N_{\mathrm{a}}}$ is a block-diagonal matrix, consisting of $2 \times 2$ block matrices, given by

$\left[\mathbf{M}_{\mathrm{A}}\right]_{2 k-1: 2 k, 2 m-1: 2 m}= \begin{cases}\sum_{j \in \mathcal{N}_{\mathrm{b}}} \boldsymbol{\Upsilon}_{k j}\left(\mathbf{p}_{k}, \boldsymbol{\kappa}_{k j}, \mathbf{p}_{k}\right), & k=m \\ \mathbf{0}, & k \neq m .\end{cases}$

On the other hand, $\mathbf{M}_{\mathrm{C}} \in \mathbb{R}^{2 N_{\mathrm{a}} \times 2 N_{\mathrm{a}}}$ is also a block matrix, consisting of $2 \times 2$ block matrices, given by (30), shown at the bottom of the page.

Structure of $\mathbf{J}_{\mathrm{e}}(\mathbf{P})$ : Combining these results, we find that the EFIM in (28) can be written as

$$
\mathbf{J}_{\mathrm{e}}(\mathbf{P})=\underbrace{\left\{\mathbf{K}_{\mathrm{A}}-\mathbf{M}_{\mathrm{A}}\right\}}_{\text {from anchors }}+\underbrace{\left\{\mathbf{K}_{\mathrm{C}}-\mathbf{M}_{\mathrm{C}}\right\}}_{\text {from cooperation }}
$$

from which we obtain (12). In (12), $\mathbf{J}_{\mathrm{e}}^{\mathrm{A}}\left(\mathbf{p}_{k}\right)=\sum_{j \in \mathcal{N}_{\mathrm{b}}} \mathbf{R}_{k}\left(\mathbf{r}_{k j}\right)$ and $\mathbf{C}_{k j}=\mathbf{C}_{j k}=\mathbf{R}_{k}\left(\mathbf{r}_{k j}\right)+\mathbf{R}_{k}\left(\mathbf{r}_{j k}\right)$ in which we have introduced the RI

$$
\mathbf{R}_{k}\left(\mathbf{r}_{k j}\right)=\boldsymbol{\Phi}_{k j}\left(\mathbf{p}_{k}, \mathbf{p}_{k}\right)-\Upsilon_{k j}\left(\mathbf{p}_{k}, \kappa_{k j}, \mathbf{p}_{k}\right)
$$

Note that in the derivation, we used

$$
\boldsymbol{\Phi}_{k m}\left(\mathbf{p}_{k}, \mathbf{p}_{m}\right)=-\boldsymbol{\Phi}_{k m}\left(\mathbf{p}_{k}, \mathbf{p}_{k}\right)
$$

and

$$
\Upsilon_{k m}\left(\mathbf{p}_{k}, \kappa_{k m}, \mathbf{p}_{m}\right)=-\Upsilon_{k m}\left(\mathbf{p}_{k}, \kappa_{k m}, \mathbf{p}_{k}\right)
$$

Since $\mathbf{J}_{\mathrm{e}}(\mathbf{P})$ in (12) can be expressed in terms of the RIs $\mathbf{R}_{k}\left(\mathbf{r}_{k j}\right)$, for $k \in \mathcal{N}_{\mathrm{a}}$ and $j \in \mathcal{N}_{\mathrm{b}} \cup \mathcal{N}_{\mathrm{a}} \backslash\{k\}$, we will examine next the details of the RIs.

\section{B. Details of the RI}

We now consider the detailed expression of the $\mathrm{RI} \mathbf{R}_{k}\left(\mathbf{r}_{k j}\right)$ in (32). We first introduce

$$
\boldsymbol{\Xi}_{k j}(\mathbf{x}, \mathbf{y}) \triangleq \mathbb{E}_{\boldsymbol{\kappa}}\left\{-\frac{\partial^{2} \ln f\left(\boldsymbol{\kappa}_{k j} \mid \mathbf{p}_{k}, \mathbf{p}_{j}\right)}{\partial \mathbf{x} \partial \mathbf{y}^{\mathrm{T}}}\right\}
$$

and

$$
\boldsymbol{\Psi}_{k j} \triangleq \mathbb{E}_{\mathbf{r}, \boldsymbol{\kappa}}\left\{-\frac{\partial^{2} \ln f\left(\mathbf{r}_{k j} \mid \mathbf{p}_{k}, \mathbf{p}_{j}, \boldsymbol{\kappa}_{k j}\right)}{\partial \tilde{\boldsymbol{\kappa}}_{k j} \partial \tilde{\boldsymbol{\kappa}}_{k j}^{\mathrm{T}}}\right\}
$$

where $\tilde{\kappa}_{k j}=\left[\begin{array}{lllllll}\tau_{k j}^{(1)} & \tilde{\alpha}_{k j}^{(1)} & \tau_{k j}^{(2)} & \tilde{\alpha}_{k j}^{(2)} & \cdots & \tau_{k j}^{\left(L_{k j}\right)} & \tilde{\alpha}_{k j}^{\left(L_{k j}\right)}\end{array}\right]^{\mathrm{T}}$ with $\tilde{\alpha}_{k j}^{(l)} \triangleq \alpha_{k j}^{(l)} / c$.

From (2) and (9), we note that $d_{k j}=\left\|\mathbf{p}_{k}-\mathbf{p}_{j}\right\|$ and that $f\left(\mathbf{r}_{k j} \mid \mathbf{p}_{k}, \mathbf{p}_{j}, \boldsymbol{\kappa}_{k j}\right)$ and $f\left(\boldsymbol{\kappa}_{k j} \mid \mathbf{p}_{k}, \mathbf{p}_{j}\right)$ only depend on $\mathbf{p}_{k}, \mathbf{p}_{j}$ through $d_{k j}$. Using the chain rule, we have

$$
\boldsymbol{\Phi}_{k j}\left(\mathbf{p}_{k}, \mathbf{p}_{k}\right)=\frac{\partial d_{k j}}{\partial \mathbf{p}_{k}} \Phi_{k j}\left(d_{k j}, d_{k j}\right) \frac{\partial d_{k j}}{\partial \mathbf{p}_{k}^{T}}
$$

and

$$
\boldsymbol{\Upsilon}_{k j}\left(\mathbf{p}_{k}, \boldsymbol{\kappa}_{k j}, \mathbf{p}_{k}\right)=\frac{\partial d_{k j}}{\partial \mathbf{p}_{k}} \Upsilon_{k j}\left(d_{k j}, \boldsymbol{\kappa}_{k j}, d_{k j}\right) \frac{\partial d_{k j}}{\partial \mathbf{p}_{k}^{T}}
$$

and hence $\mathbf{R}_{k}\left(\mathbf{r}_{k j}\right)$ can be expressed as

$$
\begin{aligned}
\mathbf{R}_{k}\left(\mathbf{r}_{k j}\right)= & \Phi_{k j}\left(d_{k j}, d_{k j}\right) \mathbf{q}_{k j} \mathbf{q}_{k j}^{\mathrm{T}} \\
& -\Upsilon_{k j}\left(d_{k j}, \boldsymbol{\kappa}_{k j}, d_{k j}\right) \mathbf{q}_{k j} \mathbf{q}_{k j}^{\mathrm{T}} \\
= & \lambda_{k j} \mathbf{q}_{k j} \mathbf{q}_{k j}^{\mathrm{T}}
\end{aligned}
$$

$$
\left[\mathbf{K}_{\mathrm{C}}\right]_{2 k-1: 2 k, 2 m-1: 2 m}= \begin{cases}\sum_{j \in \mathcal{N}_{a} \backslash\{k\}}\left[\boldsymbol{\Phi}_{k j}\left(\mathbf{p}_{k}, \mathbf{p}_{k}\right)+\mathbf{\Phi}_{j k}\left(\mathbf{p}_{k}, \mathbf{p}_{k}\right)\right], & k=m \\ \boldsymbol{\Phi}_{k m}\left(\mathbf{p}_{k}, \mathbf{p}_{m}\right)+\boldsymbol{\Phi}_{m k}\left(\mathbf{p}_{k}, \mathbf{p}_{m}\right), & k \neq m\end{cases}
$$

$$
\left[\mathbf{M}_{C}\right]_{2 k-1: 2 k, 2 m-1: 2 m}= \begin{cases}\sum_{j \in \mathcal{N}_{a} \backslash\{k\}}\left[\boldsymbol{\Upsilon}_{k j}\left(\mathbf{p}_{k}, \boldsymbol{\kappa}_{k j}, \mathbf{p}_{k}\right)+\boldsymbol{\Upsilon}_{j k}\left(\mathbf{p}_{k}, \boldsymbol{\kappa}_{j k}, \mathbf{p}_{k}\right)\right], & k=m \\ \boldsymbol{\Upsilon}_{k m}\left(\mathbf{p}_{k}, \boldsymbol{\kappa}_{k m}, \mathbf{p}_{m}\right)+\mathbf{\Upsilon}_{m k}\left(\mathbf{p}_{k}, \boldsymbol{\kappa}_{m k}, \mathbf{p}_{m}\right), & k \neq m\end{cases}
$$


where $\mathbf{q}_{k j} \triangleq \partial d_{k j} / \partial \mathbf{p}_{k}=-\partial d_{k j} / \partial \mathbf{p}_{j}=\left[\begin{array}{ll}\cos \phi_{k j} & \sin \phi_{k j}\end{array}\right]^{\mathrm{T}}$, and $\lambda_{k j}$ is given by (35), shown at the bottom of the page, where $\mathbf{l}_{k j} \triangleq \underbrace{\left[\begin{array}{lllll}1 & 0 & \cdots & 1 & 0\end{array}\right]^{\mathrm{T}}}_{2 L_{k j}}$.

\section{APPENDIX III}

\section{PROOF OF THEOREM 2}

Proof: When a priori channel knowledge is unavailable, we have $\Xi_{k j}\left(d_{k j}, d_{k j}\right)=0$, and $\boldsymbol{\Xi}_{k j}\left(d_{k j}, \boldsymbol{\kappa}_{k j}\right)=\mathbf{0}$. For NLOS signals, the RII in (35) becomes $\lambda_{k j}=0$ since $\boldsymbol{\Xi}_{k j}\left(\boldsymbol{\kappa}_{k j}, \boldsymbol{\kappa}_{k j}\right)=\mathbf{0}$. For LOS signals, however, after some algebra, the RII becomes

$$
\lambda_{k j}=\frac{1}{c^{2}} \mathbf{l}_{k j}^{\mathrm{T}} \mathbf{\Psi}_{k j}\left(\mathbf{\Psi}_{k j}+\boldsymbol{\Xi}_{k j}\left(\boldsymbol{\kappa}_{k j}, \boldsymbol{\kappa}_{k j}\right)\right)^{-1} \boldsymbol{\Xi}_{k j}\left(\boldsymbol{\kappa}_{k j}, \boldsymbol{\kappa}_{k j}\right) \mathbf{l}_{k j}
$$

where $\boldsymbol{\Xi}_{k j}\left(\boldsymbol{\kappa}_{k j}, \boldsymbol{\kappa}_{k j}\right)=\lim _{t^{2} \rightarrow \infty} \operatorname{diag}\left\{t^{2}, \mathbf{0}\right\}$ since the Fisher information for known $b_{k j}^{(1)}=0$ is infinity. To simplify (36), we partition $\boldsymbol{\Psi}_{k j}$ as

$$
\boldsymbol{\Psi}_{k j}=\left[\begin{array}{ll}
u_{k j}^{2} & \mathbf{k}_{k j}^{\mathrm{T}} \\
\mathbf{k}_{k j} & \breve{\mathbf{\Psi}}_{k j}
\end{array}\right]
$$

where $u_{k j}^{2}=8 \pi^{2} \beta^{2} \mathrm{SNR}_{k j}^{(1)}$ obtained from (33) through some algebra. As $t^{2} \rightarrow \infty$ in (36), we have

$$
\lambda_{k j}=\frac{8 \pi^{2} \beta^{2}}{c^{2}}\left(1-\chi_{k j}\right) \operatorname{SNR}_{k j}^{(1)}
$$

where

$$
\chi_{k j} \triangleq \frac{\mathbf{k}_{k j}^{\mathrm{T}} \breve{\mathbf{\Psi}}_{k j}^{-1} \mathbf{k}_{k j}}{8 \pi^{2} \beta^{2} \mathrm{SNR}_{k j}^{(1)}}
$$

is called path-overlap coefficient [29].

We next show that only the first contiguous cluster contains information for localization. Let us focus on $\chi_{k j}$. If the length of the first contiguous cluster in the received waveform is $\tilde{L}_{k j}$, where $1 \leq \tilde{L}_{k j} \leq L_{k j}$, we have [29]

$$
\mathbf{k}_{k j} \triangleq\left[\begin{array}{ll}
\tilde{\mathbf{k}}_{k j}^{\mathrm{T}} & \mathbf{0}^{\mathrm{T}}
\end{array}\right]^{\mathrm{T}} \quad \text { and } \quad \breve{\mathbf{\Psi}}_{k j} \triangleq\left[\begin{array}{cc}
\tilde{\mathbf{\Psi}}_{k j} & \mathbf{0} \\
\mathbf{0} & \boldsymbol{\nabla}
\end{array}\right]
$$

where $\tilde{\mathbf{k}}_{k j} \in \mathbb{R}^{2 \tilde{L}_{k j}-1}, \tilde{\mathbf{\Psi}}_{k j} \in \mathbb{R}^{\left(2 \tilde{L}_{k j}-1\right) \times\left(2 \tilde{L}_{k j}-1\right)}$, and $\bigotimes$ is a block matrix that is irrelevant to the rest of the derivation. Hence, (37) becomes

$$
\chi_{k j}=\frac{\tilde{\mathbf{k}}_{k j}^{\mathrm{T}} \tilde{\mathbf{\Psi}}_{k j}^{-1} \tilde{\mathbf{k}}_{k j}}{8 \pi^{2} \beta^{2} \mathrm{SNR}_{k j}^{(1)}}
$$

which depends only on the first $\tilde{L}_{k j}$ paths, implying that only the first contiguous cluster of LOS signals contains information for localization.

\section{APPENDIX IV}

PROOF OF THEOREM 3 AND COROLLARY 1

Proof: When the a priori knowledge of the agents' position is available, the derivation of EFIM (25) becomes

$$
\begin{aligned}
\ln f(\mathbf{r}, \boldsymbol{\theta})=\sum_{k \in \mathcal{N}_{\mathrm{a}}} \sum_{j \in \mathcal{N}_{\mathrm{b}} \cup \mathcal{N}_{\mathrm{a}} \backslash\{k\}}[ & {\left[\ln f\left(\mathbf{r}_{k j} \mid \mathbf{p}_{k}, \mathbf{p}_{j}, \boldsymbol{\kappa}_{k j}\right)\right.} \\
+ & \left.\ln f\left(\boldsymbol{\kappa}_{k j} \mid \mathbf{p}_{k}, \mathbf{p}_{j}\right)\right]+\ln f(\mathbf{P}) .
\end{aligned}
$$

Following the notations and derivations in Appendix II-A, we obtain the EFIM given by (14). This completes the proof of Theorem 3. Note that the structure of (14) is similar to that of (31) except the additional term $\boldsymbol{\Xi}_{\mathbf{P}}$.

The EFIM in (14) is applicable to general case. Note that $\mathbf{R}_{k}\left(\mathbf{r}_{k j}\right)$ in this case cannot be further simplified as that in (34) since we need to take expectation over the random parameter $\mathbf{P}$ in (32). However, when condition (16) holds for functions $\Phi_{k j}\left(d_{k j}, d_{k j}\right) \mathbf{q}_{k j} \mathbf{q}_{k j}^{\mathrm{T}}, \mathbf{q}_{k j} \boldsymbol{\Phi}_{k j}\left(d_{k j}, \mathbf{p}_{k}\right)$, and $\boldsymbol{\Phi}_{k j}\left(\boldsymbol{\kappa}_{k j}, \boldsymbol{\kappa}_{k j}\right)$, the expectations of those functions with respect to $\mathbf{P}$ can be replaced by the values of the functions at $\overline{\mathbf{P}}$. In such a case, the RI in (15) can be written as

$$
\mathbf{R}_{k}\left(\mathbf{r}_{k j}\right)=\bar{\lambda}_{k j} \mathbf{J}_{\mathrm{r}}\left(\bar{\phi}_{k j}\right)
$$

where $\bar{\lambda}_{k j}$ is the RII given in (35) evaluated at $\overline{\mathbf{P}}$, and $\bar{\phi}_{k j}$ is the angle from $\overline{\mathbf{p}}_{k}$ to $\overline{\mathbf{p}}_{j}$.

\section{APPENDIX V \\ PROOF OF THEOREM 4}

Proof: Consider a cooperative network with $N_{\mathrm{a}}$ agents, whose overall EFIM is given by (14). If agent $N_{\mathrm{a}}$ has infinite $a$ priori position knowledge, i.e., $\boldsymbol{\Xi}_{\mathbf{p}_{N_{\mathrm{a}}}}=\lim _{t^{2} \rightarrow \infty} \operatorname{diag}\left\{t^{2}, t^{2}\right\}$, then we apply the notion of EFI to eliminate the parameter vector $\mathbf{p}_{N_{\mathrm{a}}}$ in (14) and have

$$
\mathbf{J}_{\mathrm{e}}\left(\mathbf{p}_{1}, \ldots, \mathbf{p}_{N_{\mathrm{a}}-1}\right)=\left[\mathbf{J}_{\mathrm{e}}(\mathbf{P})\right]_{2\left(N_{\mathrm{a}}-1\right) \times 2\left(N_{\mathrm{a}}-1\right)}
$$

where we have used

$$
\begin{gathered}
\lim _{t^{2} \rightarrow \infty}\left(\sum_{j \in \mathcal{N}_{\mathrm{b}}} \mathbf{R}_{N_{\mathrm{a}}}\left(\mathbf{r}_{N_{\mathrm{a}}, j}\right)+\sum_{j \in \mathcal{N}_{\mathrm{a}} \backslash\left\{N_{\mathrm{a}}\right\}}\left[\mathbf{R}_{N_{\mathrm{a}}}\left(\mathbf{r}_{N_{\mathrm{a}}, j}\right)\right.\right. \\
\left.\left.+\mathbf{R}_{N_{\mathrm{a}}}\left(\mathbf{r}_{j, N_{\mathrm{a}}}\right)\right]+\left[\begin{array}{ll}
t^{2} & \\
& t^{2}
\end{array}\right]\right)^{-1}=\mathbf{0 .}
\end{gathered}
$$

Note that if we let $\mathcal{N}_{\mathrm{b}}^{\prime} \triangleq \mathcal{N}_{\mathrm{b}} \cup\left\{N_{\mathrm{a}}\right\}, \mathcal{N}_{\mathrm{a}}^{\prime} \triangleq \mathcal{N}_{\mathrm{a}} \backslash\left\{N_{\mathrm{a}}\right\}$, and $\mathbf{R}_{k}^{\prime}\left(\mathbf{r}_{k, N_{\mathrm{a}}}\right)=\mathbf{R}_{k}\left(\mathbf{r}_{N_{\mathrm{a}}, k}\right)+\mathbf{R}_{k}\left(\mathbf{r}_{k, N_{\mathrm{a}}}\right)$ for $k \in \mathcal{N}_{\mathrm{a}}^{\prime}$ in (38), the

$$
\begin{aligned}
\lambda_{k j} \triangleq \frac{1}{c^{2}}\left[\mathbf{l}_{k j}^{\mathrm{T}} \boldsymbol{\Psi}_{k j} \mathbf{l}_{k j}+c^{2} \Xi_{k j}\left(d_{k j}, d_{k j}\right)\right. & \\
& \left.\quad-\left(\mathbf{l}_{k j}^{\mathrm{T}} \boldsymbol{\Psi}_{k j}+c^{2} \boldsymbol{\Xi}_{k j}\left(d_{k j}, \boldsymbol{\kappa}_{k j}\right)\right)\left(\boldsymbol{\Psi}_{k j}+c^{2} \boldsymbol{\Xi}_{k j}\left(\boldsymbol{\kappa}_{k j}, \boldsymbol{\kappa}_{k j}\right)\right)^{-1}\left(\mathbf{l}_{k j}^{\mathrm{T}} \boldsymbol{\Psi}_{k j}+c^{2} \boldsymbol{\Xi}_{k j}\left(d_{k j}, \boldsymbol{\kappa}_{k j}\right)\right)^{\mathrm{T}}\right]
\end{aligned}
$$


structure of (38) becomes the same as that of (14), with a dimension decrease by 2 . Therefore, the new RI $\mathbf{R}_{k}^{\prime}\left(\mathbf{r}_{k, N_{\mathrm{a}}}\right)$ is fully utilizable, i.e., agent $N_{\mathrm{a}}$ with infinite a priori position knowledge is effectively an anchor.

\section{APPENDIX VI \\ PROOFS FOR SECTION IV}

\section{A. Proof of Proposition 2}

Proof: If the current coordinate system is rotated by angle $\phi$ and translated by $\mathbf{p}_{0}=\left[\begin{array}{ll}x_{0} & y_{0}\end{array}\right]^{\mathrm{T}}$, then the position of the agent in the new coordinate system is $\tilde{\mathbf{p}}=\mathbf{U}_{\phi} \mathbf{p}+\mathbf{p}_{0}$. Consequently, the EFIM for $\tilde{\mathbf{p}}$ is

$$
\begin{aligned}
\mathbf{J}_{\mathrm{e}}(\tilde{\mathbf{p}}) & =\left[\frac{\partial \mathbf{p}}{\partial \tilde{\mathbf{p}}}\right]^{\mathrm{T}} \mathbf{J}_{\mathrm{e}}(\mathbf{p})\left[\frac{\partial \mathbf{p}}{\partial \tilde{\mathbf{p}}}\right] \\
& =\mathbf{U}_{\phi}^{\mathrm{T}} \mathbf{J}_{\mathrm{e}}(\mathbf{p}) \mathbf{U}_{\phi} \cdot
\end{aligned}
$$

Due to the cyclic property of the trace operator [45], we immediately find that

$$
\mathcal{P}(\tilde{\mathbf{p}})=\operatorname{tr}\left\{\left[\mathbf{J}_{\mathrm{e}}(\tilde{\mathbf{p}})\right]^{-1}\right\}=\operatorname{tr}\left\{\left[\mathbf{J}_{\mathrm{e}}(\mathbf{p})\right]^{-1}\right\}=\mathcal{P}(\mathbf{p}) .
$$

\section{B. Proof of Proposition 4}

Proof: Without loss of generality, we focus on the first agent.

Lower Bound: Consider the EFIM $\mathbf{J}_{\mathrm{e}}^{\mathrm{L}}(\mathbf{P})$ shown in (42), shown at the bottom of the page. It can be obtained from $\mathbf{J}_{\mathrm{e}}(\mathbf{P})$ by setting all $\mathbf{C}_{k j}=0$ for $1<k, j \leq N_{\mathrm{a}}$. This EFIM corresponds to the situation where cooperation among agents 2 to $N_{\mathrm{a}}$ is completely ignored. One can show using elementary algebra that $\mathbf{J}_{\mathrm{e}}^{\mathrm{L}}(\mathbf{P}) \preceq \mathbf{J}_{\mathrm{e}}(\mathbf{P})$, which agrees with intuition since the cooperation information among agents 2 to $N_{\mathrm{a}}$ is not exploited. Applying the notion of EFI, we have the EFIM for the first agent as

$$
\begin{aligned}
\mathbf{J}_{\mathrm{e}}^{\mathrm{L}}\left(\mathbf{p}_{1}\right) & =\mathbf{J}_{\mathrm{e}}^{\mathrm{A}}\left(\mathbf{p}_{1}\right) \\
& +\sum_{j \in \mathcal{N}_{\mathrm{a}} \backslash\{1\}}\left[\mathbf{C}_{1, j}-\mathbf{C}_{1, j}\left(\mathbf{J}_{\mathrm{e}}^{\mathrm{A}}\left(\mathbf{p}_{j}\right)+\mathbf{C}_{1, j}\right)^{-1} \mathbf{C}_{1, j}\right] .
\end{aligned}
$$

Since $\mathbf{C}_{1, j}=\nu_{1, j} \mathbf{q}_{\phi_{1, j}} \mathbf{q}_{\phi_{1, j}}^{\mathrm{T}} \quad$ where $\mathbf{q}_{\phi_{1, j}} \triangleq$ $\left[\cos \phi_{1, j} \quad \sin \phi_{1, j}\right]^{\mathrm{T}}$, we can express $\mathbf{J}_{\mathrm{e}}^{\mathrm{L}}\left(\mathbf{p}_{1}\right)$ as

$$
\mathbf{J}_{\mathrm{e}}^{\mathrm{L}}\left(\mathbf{p}_{1}\right)=\mathbf{J}_{\mathrm{e}}^{\mathrm{A}}\left(\mathbf{p}_{1}\right)+\sum_{j \in \mathcal{N}_{\mathrm{a}} \backslash\{1\}} \xi_{1, j}^{\mathrm{L}} \mathbf{C}_{1, j}
$$

where $\xi_{1, j}^{\mathrm{L}} \triangleq 1-\nu_{1, j} \mathbf{q}_{\phi_{1, j}}^{\mathrm{T}}\left(\mathbf{J}_{\mathrm{e}}^{\mathrm{A}}\left(\mathbf{p}_{j}\right)+\mathbf{C}_{1, j}\right)^{-1} \mathbf{q}_{\phi_{1, j}}$. The coefficient $\xi_{1, j}^{\mathrm{L}}$ can be simplified as

$$
\begin{aligned}
\xi_{1, j}^{\mathrm{L}}= & 1-\nu_{1, j} \mathbf{q}_{\vartheta_{j}-\phi_{1, j}}^{\mathrm{T}} \\
& \cdot\left(\left[\begin{array}{cc}
\mu_{j} & \\
& \eta_{j}
\end{array}\right]+\nu_{1, j} \mathbf{q}_{\vartheta_{j}-\phi_{1, j}} \mathbf{q}_{\vartheta_{j}-\phi_{1, j}}^{\mathrm{T}}\right)^{-1} \mathbf{q}_{\vartheta_{j}-\phi_{1, j}} \\
= & \frac{1}{1+\nu_{1, j} \Delta_{j}\left(\phi_{1, j}\right)}
\end{aligned}
$$

where

$$
\Delta_{j}\left(\phi_{1, j}\right)=\frac{1}{\mu_{j}} \cos ^{2}\left(\vartheta_{j}-\phi_{1, j}\right)+\frac{1}{\eta_{j}} \sin ^{2}\left(\vartheta_{j}-\phi_{1, j}\right) .
$$

Upper Bound: Consider the EFIM $\mathbf{J}_{\mathrm{e}}^{\mathrm{U}}(\mathbf{P})$ shown in (45), at the bottom of the page. It can be obtained from $\mathbf{J}_{e}(\mathbf{P})$ by doubling the diagonal elements $\mathbf{C}_{k j}$ and setting the off-diagonal elements $-\mathbf{C}_{k j}=\mathbf{0}$ for $1<k, j \leq N_{\mathrm{a}}$. One can show using elementary algebra that $\mathbf{J}_{\mathrm{e}}^{\mathrm{U}}(\mathbf{P}) \succeq \mathbf{J}_{\mathrm{e}}(\mathbf{P})$, which agrees with intuition since more cooperation information among agents 2 to $N_{\mathrm{a}}$ is assumed in (45). Applying the notion of EFI and following the similar analysis leading to (43) and (44), we obtain the EFIM for agent 1 as

$$
\mathbf{J}_{\mathrm{e}}^{\mathrm{U}}\left(\mathbf{p}_{1}\right)=\mathbf{J}_{\mathrm{e}}^{\mathrm{A}}\left(\mathbf{p}_{1}\right)+\sum_{j \in \mathcal{N}_{\mathrm{a}} \backslash\{1\}} \xi_{1, j}^{\mathrm{U}} \mathbf{C}_{1, j}
$$

where

$$
\xi_{1, j}^{\mathrm{U}}=\frac{1}{1+\nu_{1, j} \tilde{\Delta}_{j}\left(\phi_{1, j}\right)}
$$

in which

$$
\tilde{\Delta}_{j}\left(\phi_{1, j}\right)=\frac{1}{\tilde{\mu}_{j}} \cos ^{2}\left(\tilde{\vartheta}_{j}-\phi_{1, j}\right)+\frac{1}{\tilde{\eta}_{j}} \sin ^{2}\left(\tilde{\vartheta}_{j}-\phi_{1, j}\right)
$$

with $\tilde{\mu}_{j}, \tilde{\eta}_{j}$, and $\tilde{\vartheta}_{j}$ satisfying

$$
\mathbf{F}\left(\tilde{\mu}_{j}, \tilde{\eta}_{j}, \tilde{\vartheta}_{j}\right)=\mathbf{J}_{\mathrm{e}}^{\mathrm{A}}\left(\mathbf{p}_{j}\right)+\sum_{k \in \mathcal{N}_{\mathrm{a}} \backslash\{1, j\}} 2 \mathbf{C}_{j k} .
$$

$$
\mathbf{J}_{\mathrm{e}}^{\mathrm{L}}(\mathbf{P})=\left[\begin{array}{cccc}
\mathbf{J}_{\mathrm{e}}^{\mathrm{A}}\left(\mathbf{p}_{1}\right)+\sum_{j \in \mathcal{N}_{\mathrm{a}} \backslash\{1\}} \mathbf{C}_{1, j} & -\mathbf{C}_{1,2} & \cdots & -\mathbf{C}_{1, N_{\mathrm{a}}} \\
-\mathbf{C}_{1,2} & \mathbf{J}_{\mathrm{e}}^{\mathrm{A}}\left(\mathbf{p}_{2}\right)+\mathbf{C}_{1,2} & & 0 \\
\vdots & & \ddots & \\
-\mathbf{C}_{1, N_{\mathrm{a}}} & 0 & & \mathbf{J}_{\mathrm{e}}^{\mathrm{A}}\left(\mathbf{p}_{N_{\mathrm{a}}}\right)+\mathbf{C}_{1, N_{\mathrm{a}}}
\end{array}\right]
$$

$$
\begin{aligned}
& \mathbf{J}_{\mathrm{e}}^{\mathrm{U}}(\mathbf{P}) \\
& =\left[\begin{array}{cccc}
\mathbf{J}_{\mathrm{e}}^{\mathrm{A}}\left(\mathbf{p}_{1}\right)+\sum_{j \in \mathcal{N}_{\mathrm{a}} \backslash\{1\}} \mathbf{C}_{1, j} & -\mathbf{C}_{1,2} & \ldots & \\
-\mathbf{C}_{1,2} & \mathbf{J}_{\mathrm{e}}^{\mathrm{A}}\left(\mathbf{p}_{2}\right)+\mathbf{C}_{1,2}+\sum_{j \in \mathcal{N}_{\mathrm{a}} \backslash\{1,2\}} 2 \mathbf{C}_{2, j} & & \\
\vdots & & \ddots & \\
-\mathbf{C}_{1, N_{\mathrm{a}}} & 0 & & \mathbf{J}_{1, N_{\mathrm{a}}}^{\mathrm{A}}\left(\mathbf{p}_{N_{\mathrm{a}}}\right)+\mathbf{C}_{1, N_{\mathrm{a}}}+\sum_{j \in \mathcal{N}_{\mathrm{a}} \backslash\left\{1, N_{\mathrm{a}}\right\}} 2 \mathbf{C}_{N_{\mathrm{a}}, j}
\end{array}\right] .
\end{aligned}
$$




\section{APPENDIX VII \\ PROOF OF THE SCALING LAWS}

Lemma 1: Let $\phi_{i}$ 's be $N$ independent identically distributed (i.i.d.) random variables with uniform distribution over $[0,2 \pi)$. Then, for any $0<\epsilon \leq 1$, there exist an $N_{0} \in \mathbb{N}$, such that $\forall N>N_{0}$

$$
\mathbb{P}\left\{\sum_{k=1}^{N} \sum_{j=1}^{N} \sin ^{2}\left(\phi_{k}-\phi_{j}\right)<\frac{N^{2}}{32}\right\}<\epsilon .
$$

Proof: First, we note that replacing $\phi_{i}$ with $\phi_{i} \bmod \pi$ preserves the value of $\sin ^{2}\left(\phi_{k}-\phi_{j}\right)$. Hence, we can consider $\phi_{i}$ 's to be i.i.d. and uniformly distributed in $[0, \pi)$.

We order the $N \phi_{i}$ 's, such that $0 \leq \phi_{(1)} \leq \phi_{(2)} \leq \cdots \leq$ $\phi_{(N)}<\pi$. Using order statistics [50], we find that the joint pdf of the $\phi_{(i)}$ 's is

$$
f\left(\phi_{(1)}, \phi_{(2)}, \ldots, \phi_{(N)}\right)=\frac{N !}{\pi^{N}} \mathbb{1}_{\left\{0 \leq \phi_{(1)} \leq \phi_{(2)} \leq \cdots \leq \phi_{(N)}<\pi\right\}}
$$

where $\mathbb{1}$ is the indicator function. From (48), the marginal pdf of $\phi_{(k)}$ can be derived as [50]

$f_{\phi_{(k)}}(x)=\frac{1}{\pi^{N}} \frac{N !}{(k-1) !(N-k) !} x^{k-1}(\pi-x)^{N-k} \mathbb{1}_{\{0 \leq x<\pi\}}$.

Now consider a large $N=8 K$ for some integer $K$, and let $\delta \triangleq \pi / 6$. The function $f_{\phi_{(K)}}(x)$ has a maximum at $x=\pi / 8$, and is monotonically decreasing in $[\pi / 8, \pi) \supset[\delta, \pi)$. Therefore, we have

$$
\mathbb{P}\left\{\phi_{(K)}>\delta\right\} \leq(\pi-\delta) f_{\phi_{(K)}}(\delta) .
$$

Since $\lim _{K \rightarrow \infty} f_{\phi_{(K)}}(\delta)=0$, there exists $K_{1} \in \mathbb{N}$ such that $\mathbb{P}\left\{\phi_{(K)}>\delta\right\}<\epsilon / 4, \forall K>K_{1}$. Note also that

$$
\mathbb{P}\left\{\phi_{(7 K+1)}<\pi-\delta\right\} \leq(\pi-\delta) f_{\phi_{(7 K+1)}}(\pi-\delta)
$$

and hence, for the same $K_{1}, \mathbb{P}\left\{\phi_{(7 K+1)}<\pi-\delta\right\}<\epsilon / 4$, $\forall K>K_{1}$. Similar arguments show that there exists $K_{2} \in \mathbb{N}$ such that $\mathbb{P}\left\{\phi_{(3 K+1)}<\pi / 2-\delta\right\}<\epsilon / 4$ and $\mathbb{P}\left\{\phi_{(5 K)}>\right.$ $\pi / 2+\delta\}<\epsilon / 4, \forall K>K_{2}$.

Combining the above results, we have with a probability $1-\epsilon$

$$
\phi_{(j)} \in \begin{cases}{[0, \delta],} & j=1, \ldots, K \\ {[\pi / 2-\delta, \pi / 2+\delta],} & j=3 K+1, \ldots, 5 K \\ {[\pi-\delta, \pi),} & j=7 K+1, \ldots, N\end{cases}
$$

when $K>\max \left\{K_{1}, K_{2}\right\}$. Therefore

$$
\begin{aligned}
\sum_{k=1}^{N} & \sum_{j=k+1}^{N} \sin ^{2}\left(\phi_{(k)}-\phi_{(j)}\right) \\
\geq & \sum_{k=1}^{K} \sum_{j=3 K+1}^{5 K} \sin ^{2}\left(\phi_{(k)}-\phi_{(j)}\right) \\
& +\sum_{k=3 K+1}^{5 K} \sum_{j=7 K+1}^{8 K} \sin ^{2}\left(\phi_{(k)}-\phi_{(j)}\right) \\
\geq & \left(\sum_{k=1}^{K} \sum_{j=3 K+1}^{5 K} 1+\sum_{k=3 K+1}^{5 K} \sum_{j=7 K+1}^{8 K} 1\right) \sin ^{2}\left(\frac{\pi}{2}-2 \delta\right) \\
= & K^{2}
\end{aligned}
$$

where ${ }^{p}$ denotes an inequality with probability approaching one as $K \rightarrow \infty$. Substituting $N=8 K$, and noting that the summation in (50) considers only half the terms (with $j>k$ ), we arrive at (47).

Moreover, the probability in (49) decreases exponentially with $K$, because if letting $a_{K} \triangleq f_{\phi_{(K)}}(\delta)$

$$
\begin{aligned}
\lim _{K \rightarrow \infty} \frac{a_{K+1}}{a_{K}} \\
=\frac{(8 K+8)(8 K+7) \cdots(8 K+1)}{(7 K+7)(7 K+6) \cdots(7 K+1) K} \frac{1}{6}\left(\frac{5}{6}\right)^{7} \\
\quad<1
\end{aligned}
$$

and hence one can see that $\epsilon$ in (47) decreases exponentially with $N$.

Lemma 2: Let $\lambda_{i}$ 's be $N$ i.i.d. random variables with arbitrary distribution on the support $\left[0, \lambda_{\max }\right]$. If $\mathbb{P}\left\{\lambda_{i} \leq \lambda_{0}\right\} \leq \epsilon<1 / 2$ for some $\lambda_{0} \in\left[0, \lambda_{\max }\right]$, then

$$
\mathbb{P}\left\{\lambda_{(N / 2+1)} \leq \lambda_{0}\right\}<\tilde{\epsilon}^{N}
$$

where $\lambda_{(i)}$ is the order statistics of $\lambda_{i}$ such that $0 \leq \lambda_{(1)} \leq$ $\lambda_{(2)} \leq \cdots \leq \lambda_{(N)}$, and $\tilde{\epsilon}=\sqrt{4 \epsilon(1-\epsilon)}$.

Proof: Denote the probability density and distribution of $\lambda_{i}$ by $f_{\lambda}$ and $F_{\lambda}$, respectively. Consider $N=2 K$ for some integer $K$ and $x \in\left[0, \lambda_{\max }\right]$ such that $F_{\lambda}(x)<1 / 2$. Using the order statistics, we have

$$
\begin{aligned}
F_{\lambda_{(K+1)}}(x) & =\sum_{j=K+1}^{N}\left(\begin{array}{c}
N \\
j
\end{array}\right) F_{\lambda}(x)^{j}\left(1-F_{\lambda}(x)\right)^{N-j} \\
& \leq \sum_{j=K+1}^{N} 2^{N} F_{\lambda}(x)^{j}\left(1-F_{\lambda}(x)\right)^{N-j} \\
& <2^{N}\left(1-F_{\lambda}(x)\right)^{N} \sum_{j=K+1}^{\infty}\left(\frac{F_{\lambda}(x)}{1-F_{\lambda}(x)}\right)^{j} \\
& =\frac{F_{\lambda}(x)}{1-F_{\lambda}(x)}\left[4 F_{\lambda}(x)\left(1-F_{\lambda}(x)\right)\right]^{K} \\
& <\left(\sqrt{4 F_{\lambda}(x)\left(1-F_{\lambda}(x)\right)}\right)^{N}
\end{aligned}
$$

where the first inequality follows from $\left(\begin{array}{c}N \\ j\end{array}\right) \leq 2^{N}$, the second inequality is due to the extension of finite summation, and the last inequality follows from $F_{\lambda}(x)<1 / 2$. Replacing $x$ with $\lambda_{0}$ gives (52).

\section{A. Proof of Theorem 5}

Proof: We consider first the noncooperative case, followed by the cooperative case. In either case, without loss of generality, we focus on the first agent at position $\mathbf{p}_{1}$.

Noncooperative Case: We will show that $\mathcal{P}\left(\mathbf{p}_{1}\right) \in \Omega\left(1 / N_{\mathrm{b}}\right)$ and $\mathcal{P}\left(\mathbf{p}_{1}\right) \in O\left(1 / N_{\mathrm{b}}\right),{ }^{18}$ which implies that $\mathcal{P}\left(\mathbf{p}_{1}\right) \in$ $\Theta\left(1 / N_{\mathrm{b}}\right)$.

${ }^{18}$ Similar to the definition of notation $\Theta(f(n))$, the notation $g(n) \in$ $\Omega(f(n))$ and $g(n) \in O(f(n))$ denote, respectively, that $g(n)$ is bounded below by $c_{1} f(n)$ and above by $c_{2} f(n)$ with probability approaching one as $n \rightarrow \infty$, for some constant $c_{1}$ and $c_{2}$. 
For an amplitude loss exponent $b$, signal powers decay with the distance following $\operatorname{SNR}(r) \propto 1 / r^{2 b}$. We can express the RII from a node at distance $r$ as

$$
\lambda(r)=\frac{Z}{r^{2 b}} \mathbb{1}_{\left\{r_{0} \leq r \leq r_{\max }\right\}}
$$

where $r_{0}$ is the minimum distance between nodes determined by the node's physical size, $r_{\max }$ is the maximum distance between nodes determined by the fixed area associated with dense network setting, and random variable $Z$ accounts for the largeand small-scale fading. Since $0 \leq Z \leq z_{1}$ for some $z_{1} \in \mathbb{R}^{+}$, there exists $z_{0} \in\left(0, z_{1}\right)$ such that $\mathbb{P}\left\{Z \leq z_{0}\right\} \leq \epsilon_{z}$ for a given $\epsilon_{z} \in(0,1)$. Thus, the RII from the $j$ th anchor is bounded as $0<\lambda_{\min } \leq \lambda_{1, j} \leq \lambda_{\max }$ with probability

$$
\mathbb{P}\left\{\lambda_{\min } \leq \lambda_{1, j} \leq \lambda_{\max }\right\} \leq 1-\epsilon_{z}
$$

where $\lambda_{\min }=z_{0} / r_{\max }^{2 b}$ and $\lambda_{\max }=z_{1} / r_{0}^{2 b}$.

On the one hand, we have

$$
\mathbf{J}_{\mathrm{e}}\left(\mathbf{p}_{1}\right) \preceq \lambda_{\max } \sum_{j \in \mathcal{N}_{\mathrm{b}}} \mathbf{J}_{\mathrm{r}}\left(\phi_{1, j}\right) .
$$

By the Cauchy-Schwarz inequality, we have

$$
\operatorname{tr}\left\{\left[\mathbf{J}_{\mathrm{e}}\left(\mathbf{p}_{1}\right)\right]^{-1}\right\} \cdot \operatorname{tr}\left\{\mathbf{J}_{\mathrm{e}}\left(\mathbf{p}_{1}\right)\right\} \geq 4 .
$$

Since the inequality (53) together with the fact that $\operatorname{tr}\left\{\mathbf{J}_{\mathrm{r}}\left(\phi_{1, j}\right)\right\}=1$ imply that $\operatorname{tr}\left\{\mathbf{J}_{\mathrm{e}}\left(\mathbf{p}_{1}\right)\right\} \leq \lambda_{\max } N_{\mathrm{b}}$, we have that

$$
\mathcal{P}\left(\mathbf{p}_{1}\right)=\operatorname{tr}\left\{\left[\mathbf{J}_{\mathrm{e}}\left(\mathbf{p}_{1}\right)\right]\right\}^{-1} \geq 4 /\left(\lambda_{\max } N_{\mathrm{b}}\right) .
$$

Therefore, $\mathcal{P}\left(\mathbf{p}_{1}\right) \in \Omega\left(1 / N_{\mathrm{b}}\right)$.

On the other hand, for the lower bound, we first order the $N_{\mathrm{b}}$ RII $\lambda_{1, j}$ 's, and then the probability of $\lambda_{\left(N_{\mathrm{b}} / 2+1\right)} \leq \lambda_{\min }$ is exponentially small by Lemma 2 , i.e.,

$$
\mathbb{P}\left\{\lambda_{\left(N_{\mathrm{b}} / 2+1\right)} \leq \lambda_{\min }\right\} \leq \tilde{\epsilon}^{N_{\mathrm{b}}}
$$

for some constant $\tilde{\epsilon} \in(0,1)$. Let $\mathcal{N}_{\mathrm{b}}^{\prime}$ denote the set of anchors with RII $\lambda_{(j)}$ such that $j \geq N_{\mathrm{b}} / 2+1$, and we have that

$$
\mathbb{P}\left\{\lambda_{\min } \sum_{j \in \mathcal{N}_{\mathrm{b}}^{\prime}} \mathbf{J}_{\mathrm{r}}\left(\phi_{1, j}\right) \preceq \mathbf{J}_{\mathrm{e}}\left(\mathbf{p}_{1}\right)\right\} \geq 1-\epsilon_{1}
$$

where the outage probability $\epsilon_{1}$ decreases exponentially with $N_{\mathrm{b}}$. Moreover, since

$$
\operatorname{tr}\left\{\left[\sum_{j \in \mathcal{N}_{\mathrm{b}}^{\prime}} \mathbf{J}_{\mathrm{r}}\left(\phi_{1, j}\right)\right]^{-1}\right\}=\frac{2 N_{\mathrm{b}} / 2}{\sum_{k \in \mathcal{N}_{\mathrm{b}}^{\prime}} \sum_{j \in \mathcal{N}_{\mathrm{b}}^{\prime}} \sin ^{2}\left(\phi_{1, k}-\phi_{1, j}\right)}
$$

applying Lemma 1 gives

$$
\mathbb{P}\left(\frac{1}{\lambda_{\min }} \operatorname{tr}\left\{\left[\sum_{j \in \mathcal{N}_{\mathrm{b}}^{\prime}} \mathbf{J}_{\mathrm{r}}\left(\phi_{1, j}\right)\right]^{-1}\right\} \leq \frac{128}{\lambda_{\min } N_{\mathrm{b}}}\right) \geq 1-\epsilon_{2}
$$

for sufficiently large $N_{\mathrm{b}}$. The inequality in (55) implies that

$$
\mathcal{P}\left(\mathbf{p}_{1}\right) \leq \frac{1}{\lambda_{\min }} \operatorname{tr}\left\{\left[\sum_{j \in \mathcal{N}_{\mathrm{b}}^{\prime}} \mathbf{J}_{\mathrm{r}}\left(\phi_{1, j}\right)\right]^{-1}\right\}
$$

and hence $\mathcal{P}\left(\mathbf{p}_{1}\right) \leq 128 /\left(\lambda_{\min } N_{\mathrm{b}}\right)$ with probability approaching one as $N_{\mathrm{b}} \rightarrow \infty$. Therefore, $\mathcal{P}\left(\mathbf{p}_{1}\right) \in O\left(1 / N_{\mathrm{b}}\right)$ with probability 1 .

Note that since both the outage probability $\epsilon_{1}$ in (55) and $\epsilon_{2}$ in (57) decrease exponentially with $N_{\mathrm{b}}$, the outage probability $\epsilon\left(N_{\mathrm{b}}\right)$ of the scaling law in (24) decreases exponentially with $N_{\mathrm{b}}$.

Cooperative Case: For the cooperative case, we will use the lower and upper approximations of the EFIM from (22) and (23). The upper approximation gives

$$
\begin{aligned}
\mathbf{J}_{\mathrm{e}}^{\mathrm{U}}\left(\mathbf{p}_{1}\right) & =\mathbf{J}_{\mathrm{e}}^{\mathrm{A}}\left(\mathbf{p}_{1}\right)+\sum_{j \in \mathcal{N}_{\mathrm{a}} \backslash\{1\}} \xi_{1, j}^{\mathrm{U}} \mathbf{C}_{1, j} \\
& \preceq \sum_{j \in \mathcal{N}_{\mathrm{b}} \cup \mathcal{N}_{\mathrm{a}} \backslash\{1\}} \lambda_{1, j} \mathbf{J}_{\mathrm{r}}\left(\phi_{1, j}\right)
\end{aligned}
$$

where the inequality is obtained by treating all other agents to be anchors, i.e., $\xi_{1, j}^{\mathrm{U}}=1\left(j \in \mathcal{N}_{\mathrm{a}}\right)$. In this case, there are equivalently $N_{\mathrm{b}}+N_{\mathrm{a}}-1$ anchors, and similar analysis as in the noncooperative case shows that $\mathcal{P}\left(\mathbf{p}_{1}\right) \in \Omega\left(1 /\left(N_{\mathrm{b}}+N_{\mathrm{a}}\right)\right)$.

On the other hand, from the lower approximation, we have, with probability approaching one, that

$$
\begin{aligned}
\mathbf{J}_{\mathrm{e}}^{\mathrm{L}}\left(\mathbf{p}_{1}\right) & =\mathbf{J}_{\mathrm{e}}^{\mathrm{A}}\left(\mathbf{p}_{1}\right)+\sum_{j \in \mathcal{N}_{\mathrm{a}} \backslash\{1\}} \xi_{1, j}^{\mathrm{L}} \mathbf{C}_{1, j} \\
& \succeq \tilde{\nu} \sum_{j \in \mathcal{N}_{\mathrm{b}} \cup \mathcal{N}_{\mathrm{a}} \backslash\{1\}} \mathbf{J}_{\mathrm{r}}\left(\phi_{1, j}\right)
\end{aligned}
$$

where $\tilde{\nu}>0$ is a given lower bound on both the RII $\lambda_{1, j}(j \in$ $\left.\mathcal{N}_{\mathrm{b}}\right)$ and the effective RII $\xi_{1, j}^{\mathrm{L}} \nu_{1, j}\left(j \in \mathcal{N}_{\mathrm{a}}\right)$. From Lemma 2, we can find such $\tilde{\nu}$ for the dense network setting, because there exist constants $0<c_{1}, c_{2}<+\infty$ such that $\lambda_{1, j}>c_{1}, \nu_{1, j}>c_{1}$, and $\Delta_{j}\left(\phi_{1, j}\right)<c_{2}$ with probability approaching one; defining $\tilde{\nu} \triangleq$ $c_{1} /\left(1+c_{1} \cdot c_{2}\right)$ implies $\lambda_{1, j} \geq \tilde{\nu}$ and $\xi_{1, j}^{\mathrm{L}} \nu_{1, j} \geq \tilde{\nu}$ since $\xi_{1, j}^{\mathrm{L}}=$ $\left[1+\Delta_{j}\left(\phi_{1, j}\right) \nu_{1, j}\right]^{-1}$. Applying Lemmas 1 and 2 , and following a similar line of reasoning as in the noncooperative case, we find $\mathcal{P}\left(\mathbf{p}_{1}\right) \in O\left(1 /\left(N_{\mathrm{b}}+N_{\mathrm{a}}\right)\right)$ with probability approaching one as $N_{\mathrm{b}}, N_{\mathrm{a}} \rightarrow+\infty$. Thus, we conclude that the SPEB in cooperative networks scales as $\Theta\left(1 /\left(N_{\mathrm{b}}+N_{\mathrm{a}}\right)\right)$.

\section{B. Proof of Theorem 6}

Proof: Let $\rho_{\mathrm{b}}$ denote the density of anchor nodes uniformly distributed in an extended network. Consider an area within distance $R$ to agent 1 , then the expected number of anchors within that area is $N_{\mathrm{b}}=\rho_{\mathrm{b}} \pi R^{2}$. Following a similar analysis leading to (54), we can show that the effect of large- and small-scale fading together with path loss on the RII can be bounded as $c_{1} / r^{2 b} \leq \lambda(r) \leq c_{2} / r^{2 b}$ for some constants $0<c_{1}<c_{2}<$ $+\infty$, with an outage probability exponentially decreasing with $N_{\mathrm{b}}$ and $N_{\mathrm{a}}$. This implies that, with probability approaching one, 
the large- and small-scale fading will not affect the scaling law, ${ }^{19}$ and hence we can consider the RII from a node at distance $r$ as

$$
\lambda(r)=\frac{1}{r^{2 b}} \mathbb{1}_{\left\{r \geq r_{0}\right\}}
$$

for the analysis of the scaling laws. Since each anchor is uniformly distributed in the given area, the pdf of the RII can be written as

$$
f(\lambda)=\frac{1}{b\left(R^{2}-r_{0}^{2}\right)} \lambda^{-\frac{b+1}{b}} \mathbb{1}_{\left\{1 / R^{2 b} \leq \lambda \leq 1 / r_{0}^{2 b}\right\}}
$$

with mean

$$
\mathbb{E}\{\lambda\}= \begin{cases}\frac{1}{b-1} \frac{r_{0}^{2-2 b}-R^{2-2 b}}{R^{2}-r_{0}^{2}}, & b>1 \\ \frac{\ln R^{2}-\ln r_{0}^{2}}{R^{2}-r_{0}^{2}}, & b=1 \\ \frac{1}{1-b} \frac{R^{2-2 b}-r_{0}^{2-2 b}}{R^{2}-r_{0}^{2}}, & 0<b<1\end{cases}
$$

and second moment

$$
\mathbb{E}\left\{\lambda^{2}\right\}= \begin{cases}\frac{1}{2 b-1} \frac{r_{0}^{2-4 b}-R^{2-4 b}}{R^{2}-r_{0}^{2}}, & b>1 \\ \frac{1}{r_{0}^{2} R^{2}}, & b=1 \\ \frac{1}{2 b-1} \frac{r_{0}^{2-4 b}-R^{2-4 b}}{R^{2}-r_{0}^{2}}, & 0<b<1 .\end{cases}
$$

Note that $N_{\mathrm{b}} \propto R^{2}$, we can show that the mean scales as

$$
\mathbb{E}\{\lambda\} \in \begin{cases}\Theta\left(1 / N_{\mathrm{b}}\right), & b>1 \\ \Theta\left(\log N_{\mathrm{b}} / N_{\mathrm{b}}\right), & b=1 \\ \Theta\left(1 / N_{\mathrm{b}}^{b}\right), & 0<b<1\end{cases}
$$

and the variance always scales as

$$
\operatorname{Var}\{\lambda\} \in \Theta\left(1 / N_{\mathrm{b}}\right)
$$

When $b>1$, it follows that, for fixed densities of anchors and agents, $\operatorname{tr}\left\{\mathbf{J}_{\mathrm{e}}\left(\mathbf{p}_{1}\right)\right\} \in \Theta(1)$ with probability approaching one as $N_{\mathrm{b}} \rightarrow+\infty$, which implies that $\mathcal{P}\left(\mathbf{p}_{1}\right) \in \Theta(1)$.

We will show that when $b=1$, the $\mathcal{P}\left(\mathbf{p}_{1}\right)$ scales as $\Theta\left(1 / \log N_{\mathrm{b}}\right)$ and $\Theta\left(1 / \log \left(N_{\mathrm{b}}+N_{\mathrm{a}}\right)\right)$ for the noncooperative case and cooperative case, respectively. Using a similar argument, we can easily show that for $0<b<1$ the SPEB scales as $\Theta\left(1 / N_{\mathrm{b}}^{b-1}\right)$ and $\Theta\left(1 /\left(N_{\mathrm{b}}+N_{\mathrm{a}}\right)^{b-1}\right)$ for the noncooperative case and cooperative case, respectively.

Noncooperative Case $(b=1)$ : We introduce a random variable $Y_{N_{\mathrm{b}}}=\sum_{j \in \mathcal{N}_{\mathrm{b}}} \lambda_{1, j} / \log \left(N_{\mathrm{b}}\right)$. From (61) and (62), we have

$$
\lim _{N_{\mathrm{b}} \rightarrow \infty} \mathbb{E}\left\{Y_{N_{\mathrm{b}}}\right\}=C
$$

\footnotetext{
${ }^{19}$ It will be shown that the overall outage is dominated by the spatial topology for a large number of nodes, and thus we can ignore the outage due to fading.
}

for some constant $C$, and

$$
\begin{aligned}
\lim _{N_{\mathrm{b}} \rightarrow \infty} & \mathbb{E}\left\{\left|Y_{N_{\mathrm{b}}}-C\right|^{2}\right\} \\
= & \lim _{N_{\mathrm{b}} \rightarrow \infty} \operatorname{Var}\left\{Y_{N_{\mathrm{b}}}\right\}+\lim _{N_{\mathrm{b}} \rightarrow \infty}\left|\mathbb{E}\left\{Y_{N_{\mathrm{b}}}\right\}-C\right|^{2} \\
& +\lim _{N_{\mathrm{b}} \rightarrow \infty} 2\left(\mathbb{E}\left\{Y_{N_{\mathrm{b}}}\right\}-C\right) \cdot \mathbb{E}\left\{Y_{N_{\mathrm{b}}}-\mathbb{E}\left\{Y_{N_{\mathrm{b}}}\right\}\right\} \\
= & 0 .
\end{aligned}
$$

This implies that $\sum_{j \in \mathcal{N}_{\mathrm{b}}} \lambda_{1, j}$ scales as $\Theta\left(\log N_{\mathrm{b}}\right)$ with probability approaching one, and hence $\operatorname{tr}\left\{\mathbf{J}_{\mathrm{e}}\left(\mathbf{p}_{1}\right)\right\} \in \Theta\left(\log N_{\mathrm{b}}\right)$. Using a similar analysis as in Appendix VII-A, we can show that $\mathcal{P}\left(\mathbf{p}_{1}\right) \in \Omega\left(1 / \log N_{\mathrm{b}}\right)$.

For the upper bound, using the same argument as in Lemma 1 , we can show that with probability approaching one, there are $N_{\mathrm{b}} / 8$ anchors with angle $\phi_{k} \in[0, \pi / 6]$ and $N_{\mathrm{b}} / 8$ anchors with angle $\phi_{k} \in[\pi / 3, \pi / 2]$ to the agent. We denote these two disjoint sets of anchors by $\mathcal{N}_{1}$ and $\mathcal{N}_{2}$, and define

$$
\tilde{\mathbf{J}}_{\mathrm{e}}\left(\mathbf{p}_{1}\right) \triangleq \sum_{j \in \mathcal{N}_{1} \cup \mathcal{N}_{2}} \lambda_{1, j} \mathbf{J}_{\mathrm{r}}\left(\phi_{1, j}\right)
$$

and

$$
\tilde{\mathbf{J}}_{\mathrm{e}}^{*}\left(\mathbf{p}_{1}\right) \triangleq\left(\sum_{j \in \mathcal{N}_{1}} \lambda_{1, j}\right) \mathbf{J}_{\mathrm{r}}(\pi / 6)+\left(\sum_{j \in \mathcal{N}_{2}} \lambda_{1, j}\right) \mathbf{J}_{\mathrm{r}}(\pi / 3) .
$$

Then, we have

$$
\begin{aligned}
\operatorname{tr}\left\{\left[\mathbf{J}_{\mathrm{e}}\left(\mathbf{p}_{1}\right)\right]^{-1}\right\} & \leq \operatorname{tr}\left\{\left[\tilde{\mathbf{J}}_{\mathrm{e}}\left(\mathbf{p}_{1}\right)\right]^{-1}\right\} \\
& \leq \operatorname{tr}\left\{\left[\tilde{\mathbf{J}}_{\mathrm{e}}^{*}\left(\mathbf{p}_{1}\right)\right]^{-1}\right\}
\end{aligned}
$$

where the first inequality comes from $\mathcal{N}_{1} \cup \mathcal{N}_{2} \subseteq \mathcal{N}_{\mathrm{b}}$, and the second inequality is due to the fact that the SPEB increases if we set $\phi_{1, j}=\pi / 6$ for $j \in \mathcal{N}_{1}$ and $\phi_{1, j}=\pi / 3$ for $j \in \mathcal{N}_{2} \cdot{ }^{20}$ Since both $\sum_{j \in \mathcal{N}_{1}} \lambda_{1, j}$ and $\sum_{j \in \mathcal{N}_{2}} \lambda_{1, j}$ scale as $\Theta\left(\log \mathcal{N}_{\mathrm{b}}\right)$, $\mathcal{P}(\mathbf{p}) \in O\left(1 / \log N_{\mathrm{b}}\right)$ with probability approaching one. Therefore, the SPEB in noncooperative extended networks scales as $\Theta\left(1 / \log N_{\mathrm{b}}\right)$.

We finally check the probability of outage, i.e., $\sum_{j \in \mathcal{N}_{\mathrm{b}}} \lambda_{1, j}$ is not in $\Theta\left(\log N_{\mathrm{b}}\right)$. For a fixed large $N_{\mathrm{b}}$, the distribution of $\sum_{j \in N_{\mathrm{b}}} \lambda_{1, j} / \sqrt{N_{\mathrm{b}}}$ can be approximated as the normal distribution $N\left(\log N_{\mathrm{b}} / \sqrt{N_{\mathrm{b}}}, 1 / N_{\mathrm{b}}\right)$, and hence 21

$$
\begin{aligned}
& \mathbb{P}\left(\left|\sum_{j \in \mathcal{N}_{\mathrm{b}}} \lambda_{1, j}-\log N_{\mathrm{b}}\right|>\frac{1}{2} \log N_{\mathrm{b}}\right) \\
& =2 Q\left(\frac{1}{2} \log N_{\mathrm{b}}\right) \\
& \cong \frac{1}{\log N_{\mathrm{b}}} \exp \left\{-\frac{1}{8} \log ^{2} N_{\mathrm{b}}\right\}
\end{aligned}
$$

where $Q(\cdot)$ is the tail probability function of standard normal distribution. Approximations and bounds for the tail probability

\footnotetext{
${ }^{20}$ This can be seen from (56) that every element in the sum of the denominator decreases if letting $\phi_{1, j}=\pi / 6$ for $j \in \mathcal{N}_{1}$ and $\phi_{1, j}=\pi / 3$ for $j \in \mathcal{N}_{2}$.

${ }^{21}$ The notation $\cong$ denotes "on the order of."
} 
function can be found in [51]-[53]. Moreover, when $0<b<1$, a similar argument leads to

$$
\begin{aligned}
\mathbb{P} & \left(\left|\sum_{k \in \mathcal{N}_{\mathrm{b}}} \lambda_{k}-N_{\mathrm{b}}^{1-b}\right|>\frac{1}{2} N_{\mathrm{b}}^{1-b}\right) \\
& =2 Q\left(\frac{1}{2} N_{\mathrm{b}}^{1-b}\right) \\
& \cong \frac{1}{N_{\mathrm{b}}^{1-b}} \exp \left\{-\frac{1}{8} N_{\mathrm{b}}^{2-2 b}\right\} .
\end{aligned}
$$

Cooperative Case $(b=1)$ : The cooperative case can be proved similar to the above noncooperative case in conjunction with the cooperative case of Theorem 5. It turns out that the SPEB can be shown to scale as $\Omega\left(1 / \log \left(N_{\mathrm{b}}+N_{\mathrm{a}}\right)\right)$ when all other agents are considered to be anchors. We can also show that, with probability approaching one, the SPEB scales as $O\left(1 / \log \left(N_{\mathrm{b}}+N_{\mathrm{a}}\right)\right)$, using the lower approximation of the EFIM, and an argument similar to (63).

\section{REFERENCES}

[1] A. Sayed, A. Tarighat, and N. Khajehnouri, "Network-based wireless location: Challenges faced in developing techniques for accurate wireless location information," IEEE Signal Process. Mag., vol. 22, no. 4, pp. 24-40, Jul. 2005.

[2] K. Pahlavan, X. Li, and J. P. Makela, "Indoor geolocation science and technology," IEEE Commun. Mag., vol. 40, no. 2, pp. 112-118, Feb. 2002.

[3] J. J. Caffery and G. L. Stuber, "Overview of radiolocation in CDMA cellular systems," IEEE Commun. Mag., vol. 36, no. 4, pp. 38-45, Apr. 1998.

[4] C.-Y. Chong and S. P. Kumar, "Sensor networks: Evolution, opportunities, and challenges," Proc. IEEE, vol. 91, no. 8, pp. 1247-1256, Aug. 2003.

[5] N. Patwari, J. N. Ash, S. Kyperountas, A. O. Hero, III, R. L. Moses, and N. S. Correal, "Locating the nodes: Cooperative localization in wireless sensor networks," IEEE Signal Process. Mag., vol. 22, no. 4, pp. 54-69, Jul. 2005.

[6] H. Wymeersch, J. Lien, and M. Z. Win, "Cooperative localization in wireless networks," Proc. IEEE, vol. 97, no. 2, pp. 427-450, Feb. 2009.

[7] S. Gezici, Z. Tian, G. B. Giannakis, H. Kobayashi, A. F. Molisch, H. V. Poor, and Z. Sahinoglu, "Localization via ultra-wideband radios: A look at positioning aspects for future sensor networks," IEEE Signal Process. Mag., vol. 22, no. 4, pp. 70-84, Jul. 2005.

[8] D. B. Jourdan, D. Dardari, and M. Z. Win, "Position error bound for UWB localization in dense cluttered environments," IEEE Trans. Aerosp. Electron. Syst., vol. 44, no. 2, pp. 613-628, Apr. 2008.

[9] E. Kaplan, Ed., Understanding GPS: Principles and Applications. Reading, MA: Artech House, 1996.

[10] J. J. Spilker, Jr., "GPS signal structure and performance characteristics," J. Inst. Navigat., vol. 25, no. 2, pp. 121-146, Summer 1978.

[11] J. Zagami, S. Parl, J. Bussgang, and K. Melillo, "Providing universal location services using a wireless E911 location network," IEEE Commun. Mag., vol. 36, no. 4, pp. 66-71, Apr. 1998.

[12] J. Hightower, A. LaMarca, and I. Smith, "Practical lessons from place lab," IEEE Pervasive Comput., vol. 5, no. 3, pp. 32-39, Jul.-Sep. 2006.

[13] M. Z. Win and R. A. Scholtz, "Ultra-wide bandwidth time-hopping spread-spectrum impulse radio for wireless multiple-access communications," IEEE Trans. Commun., vol. 48, no. 4, pp. 679-691, Apr. 2000.

[14] D. Cassioli, M. Z. Win, and A. F. Molisch, "The ultra-wide bandwidth indoor channel: From statistical model to simulations," IEEE J. Sel. Areas Commun., vol. 20, no. 6, pp. 1247-1257, Aug. 2002.

[15] J.-Y. Lee and R. A. Scholtz, "Ranging in a dense multipath environment using an UWB radio link," IEEE J. Sel. Areas Commun., vol. 20, no. 9, pp. 1677-1683, Dec. 2002.

[16] D. Dardari, A. Conti, U. J. Ferner, A. Giorgetti, and M. Z. Win, "Ranging with ultrawide bandwidth signals in multipath environments," Proc. IEEE, vol. 97, no. 2, pp. 404-426, Feb. 2009.

[17] Z. Zhang, C. L. Law, and Y. L. Guan, "BA-POC-based ranging method with multipath mitigation," IEEE Antennas Wireless Propag. Lett., vol. 4, pp. 492-495, 2005.
[18] Z. N. Low, J. H. Cheong, C. L. Law, W. T. Ng, and Y. J. Lee, "Pulse detection algorithm for line-of-sight (LOS) UWB ranging applications," IEEE Antennas Wireless Propag. Lett., vol. 4, pp. 63-67, 2005.

[19] D. Cassioli, M. Z. Win, F. Vatalaro, and A. F. Molisch, "Low-complexity Rake receivers in ultra-wideband channels," IEEE Trans. Wireless Commun., vol. 6, no. 4, pp. 1265-1275, Apr. 2007.

[20] T. Q. S. Quek, M. Z. Win, and D. Dardari, "Unified analysis of UWB transmitted-reference schemes in the presence of narrowband interference," IEEE Trans. Wireless Commun., vol. 6, no. 6, pp. 2126-2139, Jun. 2007.

[21] L. Yang and G. B. Giannakis, "Ultra-wideband communications: An idea whose time has come," IEEE Signal Process. Mag., vol. 21, no. 6, pp. 26-54, Nov. 2004

[22] A. Ridolfi and M. Z. Win, "Ultrawide bandwidth signals as shot-noise: A unifying approach," IEEE J. Sel. Areas Commun., vol. 24, no. 4, pp. 899-905, Apr. 2006.

[23] W. Suwansantisuk and M. Z. Win, "Multipath aided rapid acquisition: Optimal search strategies," IEEE Trans. Inf. Theory, vol. 53, no. 1, pp. 174-193, Jan. 2007.

[24] A. F. Molisch, "Ultrawideband propagation channels-theory, measurements, and modeling," IEEE Trans. Veh. Technol., vol. 54, no. 5, pp. $1528-1545$, Sep. 2005.

[25] A. F. Molisch, "Ultra-wide-band propagation channels," Proc. IEEE, vol. 97, no. 2, pp. 353-371, Feb. 2009.

[26] C. Savarese, J. M. Rabaey, and J. Beutel, "Locationing in distributed ad-hoc wireless sensor networks," in Proc. IEEE Int. Conf. Acoust. Speech Signal Process., May 2001, vol. 4, no. 7-11, pp. 2037-2040.

[27] C. Chang and A. Sahai, "Estimation bounds for localization," in Proc. IEEE Conf. Sensor Ad Hoc Commun. Netw., Santa Clara, CA, Oct. 2004, pp. 415-424.

[28] E. G. Larsson, "Cramér-Rao bound analysis of distributed positioning in sensor networks," IEEE Signal Process. Lett., vol. 11, no. 3, pp. 334-337, Mar. 2004.

[29] Y. Shen and M. Z. Win, "Fundamental limits of wideband localization-Part I: A general framework," IEEE Trans. Inf. Theory, vol. 56, no. 10, Oct. 2010, DOI: 10.1109/TIT.20102060110.

[30] Y. Shen and M. Z. Win, "Localization accuracy using wideband antenna arrays," IEEE Trans. Commun., vol. 58, no. 1, pp. 270-280, Jan. 2010.

[31] Y. Qi, H. Kobayashi, and H. Suda, "Analysis of wireless geolocation in a non-line-of-sight environment," IEEE Trans. Wireless Commun., vol. 5, no. 3, pp. 672-681, Mar. 2006.

[32] L. Mailaender, "On the geolocation bounds for round-trip time-of-arrival and all non-line-of-sight channels," EURASIP J. Adv. Signal Process., vol. 2008, p. 10, 2008.

[33] J. Caffery, Ed., Wireless Location in CDMA Cellular Radio Systems. Boston, MA: Kluwer, 2000.

[34] T. Rappaport, J. Reed, and B. Woerner, "Position location using wireless communications on highways of the future," IEEE Commun. Mag., vol. 34, no. 10, pp. 33-41, Oct. 1996.

[35] D. Niculescu and B. Nath, "Ad hoc positioning system (APS) using AOA,” in Proc. IEEE Conf. Comput. Commun., Mar./Apr. 2003, vol. 3, pp. $1734-1743$.

[36] N. Patwari and A. O. Hero, III, "Using proximity and quantized RSS for sensor localization in wireless networks," in Proc. IEEE/ACM 2nd Workshop Wireless Sensor Nets. Appl., 2003, pp. 20-29.

[37] T. Pavani, G. Costa, M. Mazzotti, A. Conti, and D. Dardari, "Experimental results on indoor localization techniques through wireless sensors network," in Proc. IEEE Semiannu. Veh. Technol. Conf., May 2006, vol. 2, pp. 663-667.

[38] M. Hamilton and P. Schultheiss, "Passive ranging in multipath dominant environments. I. Known multipath parameters," IEEE Trans. Signal Process., vol. 40, no. 1, pp. 1-12, Jan. 1992.

[39] J. Lee and S. Yoo, "Large error performance of UWB ranging," in Proc. IEEE Int. Conf. Ultra-Wideband, Zurich, Switzerland, 2005, pp. 308-313.

[40] A. F. Molisch, Wireless Communications, 1st ed. Piscataway, NJ: IEEE Press/Wiley, 2005.

[41] A. A. Saleh and R. A. Valenzuela, "A statistical model for indoor multipath propagation," IEEE J. Sel. Areas Commun., vol. 5, no. 2, pp. 128-137, Feb. 1987.

[42] H. L. Van Trees, Detection, Estimation and Modulation Theory. New York: Wiley, 1968, vol. 1.

[43] H. V. Poor, An Introduction to Signal Detection and Estimation, 2nd ed. New York: Springer-Verlag, 1994.

[44] I. Reuven and H. Messer, "A Barankin-type lower bound on the estimation error of a hybrid parameter vector," IEEE Trans. Inf. Theory, vol. 43, no. 3, pp. 1084-1093, May 1997.

[45] R. A. Horn and C. R. Johnson, Matrix Analysis, 1st ed. Cambridge, U.K.: Cambridge Univ. Press, 1985. 
[46] C. Botteron, A. Host-Madsen, and M. Fattouche, "Cramer-Rao bounds for the estimation of multipath parameters and mobiles' positions in asynchronous DS-CDMA systems," IEEE Trans. Signal Process., vol. 52, no. 4, pp. 862-875, Apr. 2004.

[47] P. Gupta and P. R. Kumar, "The capacity of wireless networks," IEEE Trans. Inf. Theory, vol. 46, no. 2, pp. 388-404, Mar. 2000.

[48] A. Ozgur, O. Leveque, and D. N. C. Tse, "Hierarchical cooperation achieves optimal capacity scaling in ad hoc networks," IEEE Trans. Inf. Theory, vol. 53, no. 10, pp. 3549-3572, Oct. 2007.

[49] A. Goldsmith, Wireless Communications. Cambridge, U.K.: Cambridge Univ. Press, 2005.

[50] B. C. Arnold, N. Balakrishnan, and H. N. Nagaraja, A First Course in Order Statistics. New York: Wiley-Interscience, 1992.

[51] A. Conti, M. Z. Win, and M. Chiani, "On the inverse symbol error probability for diversity reception," IEEE Trans. Commun., vol. 51, no. 5, pp. 753-756, May 2003.

[52] G. Grimmett and D. Stirzaker, Probability and Random Processes, 3rd ed. Oxford, U.K.: Oxford Univ. Press, 2001.

[53] M. Chiani, D. Dardari, and M. K. Simon, "New exponential bounds and approximations for the computation of error probability in fading channels," IEEE Trans. Wireless Commun., vol. 2, no. 4, pp. 840-845, Jul. 2003.

Yuan Shen (S'05) received the B.S. degree in electrical engineering (with highest honor) from Tsinghua University, Beijing, China, in 2005 and the S.M. degree in electrical engineering from the Massachusetts Institute of Technology (MIT), Cambridge, in 2008, where he is currently working towards the Ph.D. degree at the Wireless Communications and Network Science Laboratory.

He was with the Hewlett-Packard Labs, in winter 2009, the Corporate R\&D of Qualcomm Inc., in summer 2008, and the Intelligent Transportation Information System Laboratory, Tsinghua University, from 2003 to 2005 . His research interests include communication theory, information theory, and statistical signal processing. His current research focuses on wideband localization, cooperative networks, and ultrawide bandwidth communications.

Mr. Shen served as a member of the Technical Program Committee (TPC) for the IEEE Global Communications Conference (GLOBECOM) in 2010, the IEEE International Conference on Communications (ICC) in 2010, and the IEEE Wireless Communications \& Networking Conference (WCNC) in 2009 and 2010. He received the Marconi Young Scholar Award from the Marconi Society in 2010, the Ernst A. Guillemin Thesis Award (first place) for the best S.M. thesis from the Department of Electrical Engineering and Computer Science at MIT in 2008, the Roberto Padovani Scholarship from Qualcomm Inc. in 2008, the Best Paper Award from the IEEE WCNC in 2007, and the Walter A. Rosenblith Presidential Fellowship from MIT in 2005.

Henk Wymeersch (S'98-M'05) received the Ph.D. degree in electrical engineering/applied sciences from Ghent University, Ghent, Belgium, in 2005.

Currently, he is an Assistant Professor with the Department of Signals and Systems, Chalmers University, Gothenburg, Sweden. Prior to joining Chalmers, he was a Postdoctoral Associate with the Laboratory for Information and Decision Systems (LIDS), Massachusetts Institute of Technology (MIT), Cambridge. He is the author of the book Iterative Receiver Design (Cambridge, U.K.: Cambridge Univ. Press, 2007). His research interests include algorithm design for wireless transmission, statistical inference, and iterative processing.
Moe Z. Win (S'85-M'87-SM'97-F'04) received both the Ph.D. degree in electrical engineering and the M.S. degree in applied mathematics as a Presidential Fellow at the University of Southern California (USC) in 1998. He received the M.S. degree in electrical engineering from USC in 1989, and the B.S. degree (magna cum laude) in electrical engineering from Texas A\&M University in 1987.

$\mathrm{He}$ is an Associate Professor at the Massachusetts Institute of Technology (MIT). Prior to joining MIT, he was at AT\&T Research Laboratories for five years and at the Jet Propulsion Laboratory for seven years. His research encompasses developing fundamental theories, designing algorithms, and conducting experimentation for a broad range of real-world problems. His current research topics include location-aware networks, intrinsically secure wireless networks, aggregate interference in heterogeneous networks, ultrawide bandwidth systems, multiple antenna systems, time-varying channels, optical transmission systems, and space communications systems.

$\mathrm{He}$ is an IEEE Distinguished Lecturer and elected Fellow of the IEEE, cited for "contributions to wideband wireless transmission." He was honored with the IEEE Eric E. Sumner Award (2006), an IEEE Technical Field Award for "pioneering contributions to ultra-wide band communications science and technology." Together with students and colleagues, his papers have received several awards including the IEEE Communications Society's Guglielmo Marconi Best Paper Award (2008) and the IEEE Antennas and Propagation Society's Sergei A. Schelkunoff Transactions Prize Paper Award (2003). His other recognitions include the Laurea Honoris Causa from the University of Ferrara, Italy (2008), the Technical Recognition Award of the IEEE ComSoc Radio Communications Committee (2008), Wireless Educator of the Year Award (2007), the Fulbright Foundation Senior Scholar Lecturing and Research Fellowship (2004), the U.S. Presidential Early Career Award for Scientists and Engineers (2004), the AIAA Young Aerospace Engineer of the Year (2004), and the Office of Naval Research Young Investigator Award (2003).

Prof. Win has been actively involved in organizing and chairing a number of international conferences. He served as the Technical Program Chair for the IEEE Wireless Communications and Networking Conference in 2009, the IEEE Conference on Ultra Wideband in 2006, the IEEE Communication Theory Symposia of ICC-2004 and Globecom-2000, and the IEEE Conference on Ultra Wideband Systems and Technologies in 2002; Technical Program Vice-Chair for the IEEE International Conference on Communications in 2002; and the Tutorial Chair for ICC-2009 and the IEEE Semiannual International Vehicular Technology Conference in Fall 2001. He was the chair (2004-2006) and secretary (2002-2004) for the Radio Communications Committee of the IEEE Communications Society. He is currently an Editor for the IEEE TRANSACTIONS ON WIRELESS COMMUNICATIONS. He served as Area Editor for Modulation and Signal Design (2003-2006), Editor for Wideband Wireless and Diversity (2003-2006), and Editor for Equalization and Diversity (1998-2003), all for the IEEE TRANSACTIONS ON COMMUNICATIONS. He was Guest-Editor for the PROCEEDINGS OF THE IEEE (Special Issue on UWB Technology and Emerging Applications) in 2009 and the IEEE JOURNAL ON SELECTED AREAS IN COMMUNICATIONS (Special Issue on Ultra-Wideband Radio in Multiaccess Wireless Communications) in 2002. 